\title{
Circadian rhythm influences induction of trained immunity by BCG vaccination
}

\author{
L. Charlotte J. de Bree, ${ }^{1,2,3}$ Vera P. Mourits, ${ }^{1}$ Valerie A.C.M. Koeken, ${ }^{1}$ Simone J.C.F.M. Moorlag, ${ }^{1}$ Robine Janssen, ${ }^{1}$ \\ Lukas Folkman, ${ }^{4}$ Daniele Barreca, ${ }^{4}$ Thomas Krausgruber, ${ }^{4}$ Victoria Fife-Gernedl, ${ }^{4}$ Boris Novakovic, ${ }^{5}$ Rob J.W. Arts, ${ }^{1}$ \\ Helga Dijkstra, ${ }^{1}$ Heidi Lemmers, ${ }^{1}$ Christoph Bock, ${ }^{4,6}$ Leo A.B. Joosten, ${ }^{1}$ Reinout van Crevel, ${ }^{1}$ Christine S. Benn, ${ }^{2,3}$ \\ and Mihai G. Netea ${ }^{1,7}$
}

'Radboud Center for Infectious Diseases and Department of Internal Medicine, Radboud University Medical Center, Nijmegen, Netherlands. 'Bandim Health Project, OPEN, Institute of Clinical Research, University of Southern Denmark/Odense University Hospital, Odense, Denmark. ${ }^{3}$ Danish Institute for Advanced Study, University of Southern Denmark, Odense, Denmark. ${ }^{4}$ CeMM Research Center for Molecular Medicine of the Austrian Academy of Sciences, Vienna, Austria. ${ }^{~}$ Epigenetics Research, Murdoch Children's Research Institute, Parkville, Australia and Department of Paediatrics, University of Melbourne, Melbourne, Australia. ${ }^{6}$ Department of Laboratory Medicine, Medical University of Vienna, Vienna, Austria. ${ }^{7}$ Department for Genomics and Immunoregulation, Life and Medical Sciences Institute (LIMES), University of Bonn, Bonn, Germany.

BACKCROUND. The antituberculosis vaccine bacillus Calmette-Guérin (BCG) reduces overall infant mortality. Induction of innate immune memory, also termed trained immunity, contributes toward protection against heterologous infections. Since immune cells display oscillations in numbers and function throughout the day, we investigated the effect of BCC administration time on the induction of trained immunity.

\begin{abstract}
METHODS. Eighteen volunteers were vaccinated with BCC at 6 pm and compared with 36 age- and sex-matched volunteers vaccinated between 8 am and 9 am. Peripheral blood mononuclear cells were stimulated with Staphylococcus aureus and Mycobacterium tuberculosis before, as well as 2 weeks and 3 months after, BCC vaccination. Cytokine production was measured to assess the induction of trained immunity and adaptive responses, respectively. Additionally, the influence of vaccination time on induction of trained immunity was studied in an independent cohort of 302 individuals vaccinated between 8 am and 12 pm with BCC.
\end{abstract}

RESULTS. Compared with evening vaccination, morning vaccination elicited both a stronger trained immunity and adaptive immune phenotype. In a large cohort of $\mathbf{3 0 2}$ volunteers, early morning vaccination resulted in a superior cytokine production capacity compared with later morning. A cellular, rather than soluble, substrate of the circadian effect of BCG vaccination was demonstrated by the enhanced capacity to induce trained immunity in vitro in morning-compared with evening-isolated monocytes.

CONCLUSIONS. BCG vaccination in the morning induces stronger trained immunity and adaptive responses compared with evening vaccination. Future studies should take vaccine administration time into account when studying specific and nonspecific effects of vaccines; early morning should be the preferred moment of BCG administration.

FUNDING. The Netherlands Organization for Scientific Research, the European Research Council, and the Danish National Research Foundation.

\section{Introduction}

The antituberculosis vaccine bacillus Calmette-Guérin (BCG) has the highest vaccine coverage worldwide (1). BCG protects primarily against disseminated tuberculosis (TB) in children (2), whereas protection against pulmonary TB in adults is modest (3,

\section{Related Commentary: p. 5121}

Authorship note: LCJDB and VPM contributed equally to this work. Conflict of interest: MCN and LABJ are scientific founders of Trained Therapeutics Discovery.

Copyright: @ 2020, American Society for Clinical Investigation.

Submitted: September 30, 2019; Accepted: July 14, 2020; Published: September 21, 2020.

Reference information: J Clin Invest. 2020;130(10):5603-5617.

https://doi.org/10.1172/JCl133934.
4). While the world is striving for a more effective anti-TB vaccine (5), there is mounting evidence that BCG possesses general immune modulatory properties (6). Epidemiological data and randomized trials have shown that BCG vaccination reduces allcause morbidity and mortality in neonates and children (7-13). In these trials, a reduced incidence of respiratory infections and sepsis, as well as in-hospital mortality, has been found.

Previously we have demonstrated that BCG vaccination induces nonspecific innate immune memory responses. This phenomenon, called trained immunity (14), has been postulated to explain, at least in part, the nonspecific beneficial effects of BCG vaccination on child morbidity and mortality. Epigenetic modification and metabolic reprogramming of monocytes are shown to be responsible for upregulated cytokine responses upon ex vivo stimulation of peripheral blood mononuclear cells (PBMCs) with 
BCG-unrelated stimuli such as Staphylococcus aureus, Candida albicans, and the Toll-like receptor 4 (TLR4) ligand lipopolysaccharide (LPS) (15-17). These longer-lasting nonspecific immunological effects, which persist up to 1 year after BCG vaccination, are explained by transcriptional changes associated with myeloid cell development and function in hematopoietic stem and progenitor cells, which are epigenetically conveyed into peripheral blood $\mathrm{CD}_{14}{ }^{+}$monocytes $(18,19)$. Induction of trained immunity has primarily been studied in monocytes $(16,20-22)$, but BCG exerts nonspecific effects on other innate immune cells such as NK cells and $\gamma \delta$ T cells as well $(15,23)$. In human challenge models, BCG vaccination reduced yellow fever vaccine viremia upon subsequent vaccination (20), and in a controlled human malaria infection model, a subset of BCG-vaccinated volunteers responded with reduced Plasmodium falciparum parasitemia, which correlated with early monocyte, NK, and $\gamma \delta$ T cell activation during bloodstream infection (23).

In multiple hematopoietic cell lineages, the importance of intrinsic circadian molecular clocks has been identified (24-26). Circadian rhythm reflects any biological process with a 24-hour rhythm that adapts to environmental changes due to the Earth's rotation. A central clock situated in the central suprachiasmatic nucleus of the hypothalamus coordinates peripheral molecular clocks present within cells located throughout the body, including immune cells (the intrinsic circadian molecular clocks). Circadian clock genes have been shown to oscillate in human PBMCs (24). Approximately $8 \%$ of the macrophage transcriptome oscillates in a circadian fashion, including many important regulators for pathogen recognition and cytokine secretion (26). A growing body of literature has acknowledged the importance of circadian rhythms in immune function (27). Relative and absolute numbers of hematopoietic stem cells and most mature leukocytes in the circulation fluctuate throughout the day (28). In addition, cellular functions such as phagocytic capacity, migration, and proliferation display circadian oscillations (29).

Although the attention to chronobiology and pharmacology is rising, little is known about the influence of circadian immune rhythms on vaccine immunogenicity. To our knowledge, previous studies have exclusively focused on the effect of timing of vaccine administration on induction of vaccine-specific antibody responses. Although results of studies focusing on influenza and hepatitis A immunogenicity hinted toward increased induction of specific immune responses when individuals were vaccinated in the morning $(30,31)$, another study found the effect to be dependent on the moment of sample collection rather than the timing of influenza vaccine administration (32).

The influence of timing of vaccine administration on nonspecific immunological effects of vaccines has not been investigated so far. Knowledge about possible oscillations in induction of BCG-induced trained immunity in vivo would be of great importance for assessing (nonspecific) vaccine efficacy, as well as for potential implications of BCG vaccination as immune modulator $(12,33,34)$. Therefore, we investigated the effect of timing of BCG administration on the induction of trained immunity, in order to assess whether the time of day should be taken into account to maximize health benefits after BCG vaccination.

\section{Results}

$B C G$-vaccinated healthy individuals. Eighteen healthy volunteers (11 females, 7 males) of Western European ancestry participating in the 300BCG cohort study were vaccinated in the evening and each individual was retrospectively age and sex matched with 2 individuals from the 300BCG cohort, who were vaccinated between 8 am and 9 am, resulting in a subgroup of 36 age- and sex-matched morning-vaccinated controls (Figure 1 and Figure $2 \mathrm{~A})$. The median age of both groups was 26 years. All volunteers developed a scar 3 months after BCG vaccination and the average scar size did not differ significantly between groups. An overview of participant characteristics is presented in Table 1.

Complete blood counts were measured in EDTA blood samples. Whole blood leukocyte, monocyte, lymphocyte, and neutrophil counts did not differ significantly between the morning- and evening-vaccinated subgroups at baseline, 2 weeks, or 3 months after BCG vaccination (Figure 2B).

$B C G$ vaccination in the morning elicits a stronger trained immunity phenotype compared with evening vaccination. BCG vaccination in the morning induced trained immunity, resulting in significantly enhanced $S$. aureus-induced interleukin $1 \beta$ (IL-1 $\beta$ ) and tumor necrosis factor $\alpha(\mathrm{TNF}-\alpha)$ production 2 weeks and/or 3 months after BCG vaccination (Figure 3, A-C). Overall, no induction of trained immunity was observed in the evening-vaccinated subgroup (Figure 3, D-F). IL-1 $\beta$ and IL-6 production after 24-hour M. tuberculosis stimulation was induced in both groups (Supplemental Figure 1; supplemental material available online with this article; https:// doi.org/10.1172/JCI133934DS1). However, a significant upregulation of IL-1 $\beta$ production upon $M$. tuberculosis stimulation 2 weeks after BCG vaccination was only apparent in the evening-vaccinated group, whereas 3 months after BCG vaccination this was only apparent in the morning-vaccinated group. M.tuberculosis-induced IL-6 production was upregulated in both groups after 2 weeks (Supplemental Figure 1). Specific M. tuberculosis IFN- $\gamma$ responses were significantly higher in morning-vaccinated individuals 3 months after BCG vaccination (Figure $3 G$ ). In contrast with BCG administration in the morning, BCG vaccination in the evening did not elicit an increased IFN- $\gamma$ response upon $M$. tuberculosis restimulation (Figure 3H). The significant changes in cytokine production upon restimulation after BCG vaccination due to timing, as shown in Figure 3, A-H, could explain between $7 \%$ and $12 \%$ of the overall variation in cytokine production within this cohort.

Changes in cytokine production after BCG vaccination were not explained by altered percentages of monocytes or lymphocytes in the PBMC fraction after Ficoll isolation, since those remained stable between time points and did not differ between groups (Figure 3I).

Trained immunity responses induced by BCG vaccination are most profound in the early morning. We subsequently assessed whether BCG administration at different time points during the morning (between $8 \mathrm{am}$ and $12 \mathrm{pm}$ ) within the entire 300BCG cohort (Figure 4A), composed of 302 (171 female and 131 male) volunteers, would affect the induction of trained immunity in terms of cytokine production. Participant characteristics of the entire 300BCG cohort can be found in Supplemental Table 1. Interestingly, significant differences in fold changes were found between the early morning-vaccinated group ( 8 am to 9 am, $n=$ 


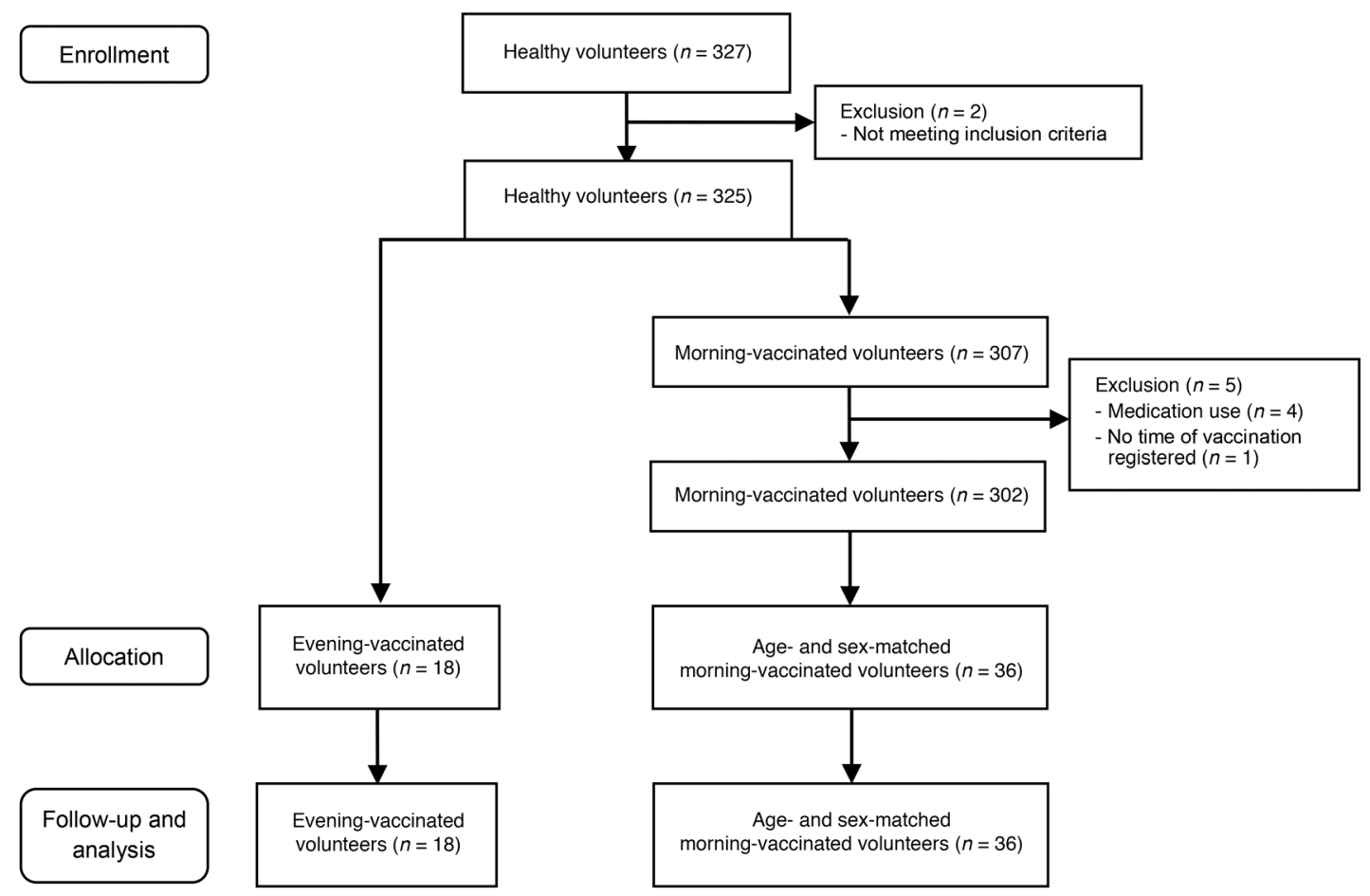

Figure 1. Flow diagram of individuals included in this study. A total of 327 healthy volunteers were assessed, of which 2 did not meet inclusion criteria. Of the 325 individuals vaccinated, 18 participants were vaccinated in the evening and 307 were vaccinated in the morning. Of the 307 morning-vaccinated individuals, 5 were excluded due to medication use or lack of information. Of the 302 morning-vaccinated individuals, 36 sex- and age-matched controls were selected for further analysis with the evening-vaccinated individuals.

68) and late morning-vaccinated group (11 am to $12 \mathrm{pm}, n=66)$ for $S$. aureus-induced IL-1 $\beta$ and TNF- $\alpha$, and between 8-9 am and 10-11 am $(n=8)$ subgroups for $S$. aureus-induced IL-6 two weeks after vaccination in favor of the early morning-vaccinated group (Figure 4, B-D). After 3 months, S. aureus-induced IL-1 $\beta$ and TNF- $\alpha$ remained significantly higher in the early morningvaccinated group (Figure 4, B-D). No significant differences were observed between different morning-vaccinated subgroups in $M$. tuberculosis-induced IFN- $\gamma$ responses 2 weeks and 3 months after vaccination (Figure $4 \mathrm{E}$ ), which was also seen for IL-1 $\beta$ and IL-6 production (Supplemental Figure 2).

In contrast with baseline $S$. aureus-induced IL- 6 and TNF- $\alpha$ responses, which did not differ between groups, baseline $S$. aureus-induced IL-1 $1 \beta$ responses were significantly higher in the late morning-vaccinated (11 am to $12 \mathrm{pm}$ ) individuals, compared

\section{Table 1. Characteristics of morning- and evening-vaccinated volunteers}

$\begin{array}{lcc} & \begin{array}{c}\text { Morning vaccinated } \\ (n=36)\end{array} & \begin{array}{c}\text { Evening vaccinated } \\ (n=18)\end{array} \\ \text { Age (years) } & 26.2(\text { SD 9.8) } & 25.8(\text { SD 10.7) } \\ \text { Sex (percentage female) } & 61 \% & 61 \% \\ \text { BMI } & 22.0(\text { SD 2.0) } & 21.9(\text { SD 2.0) } \\ \text { BCG scar size (cm) } & 0.44 \text { (SD 0.14) } & 0.41(\text { SD 0.12) } \\ \text { Smoking }(n) & 1 & 1\end{array}$

Mean \pm SD; morning vaccinated $n=36$, evening vaccinated $n=18$. with those vaccinated between 8 am and 11 am (Supplemental Figure 3, A-C). M. tuberculosis-induced IFN- $\gamma$ responses did not differ between morning subgroups (Supplemental Figure 3D). When $S$. aureus-induced IL-1 $\beta$ responses are corrected for time of blood drawing, the differences in fold changes between early morning and late morning vaccination are no longer significant (Supplemental Figure 3E). We found a modest but significant inverse correlation between percentages of lymphocytes in the isolated PMBC fraction at baseline and time of BCG administration, and a positive correlation between percentages of monocytes in isolated PBMCs at baseline and time of BCG administration (Figure 5, A and B). Thus, on average, the earlier the moment of blood collection during the morning, the higher the lymphocyte percentages and the lower the monocyte percentages. Nevertheless, fold changes of lymphocyte and monocyte percentages did not significantly differ between the different morning subgroups (Figure 5, C and D). After correction for monocyte percentages within the PBMC fraction, differences between morning-vaccinated subgroups remained apparent for the level of IL-1 $\beta$, IL- 6 , and TNF- $\alpha$ production in response to $S$. aureus stimulation (Supplemental Figure 4).

Cell-intrinsic effect of circadian rhythm on induction of trained immunity. There are 2 main mechanisms that could explain the differential induction of trained immunity by morning or evening BCG vaccination: (i) changes in circulating modulators of immune responses, and (ii) cell-intrinsic circadian changes. The most obvious candidate for circulating modulators affected by circadian rhythm are corticosteroid hormones (35). As expected, circulating cortisol levels were significantly higher in the early morning 


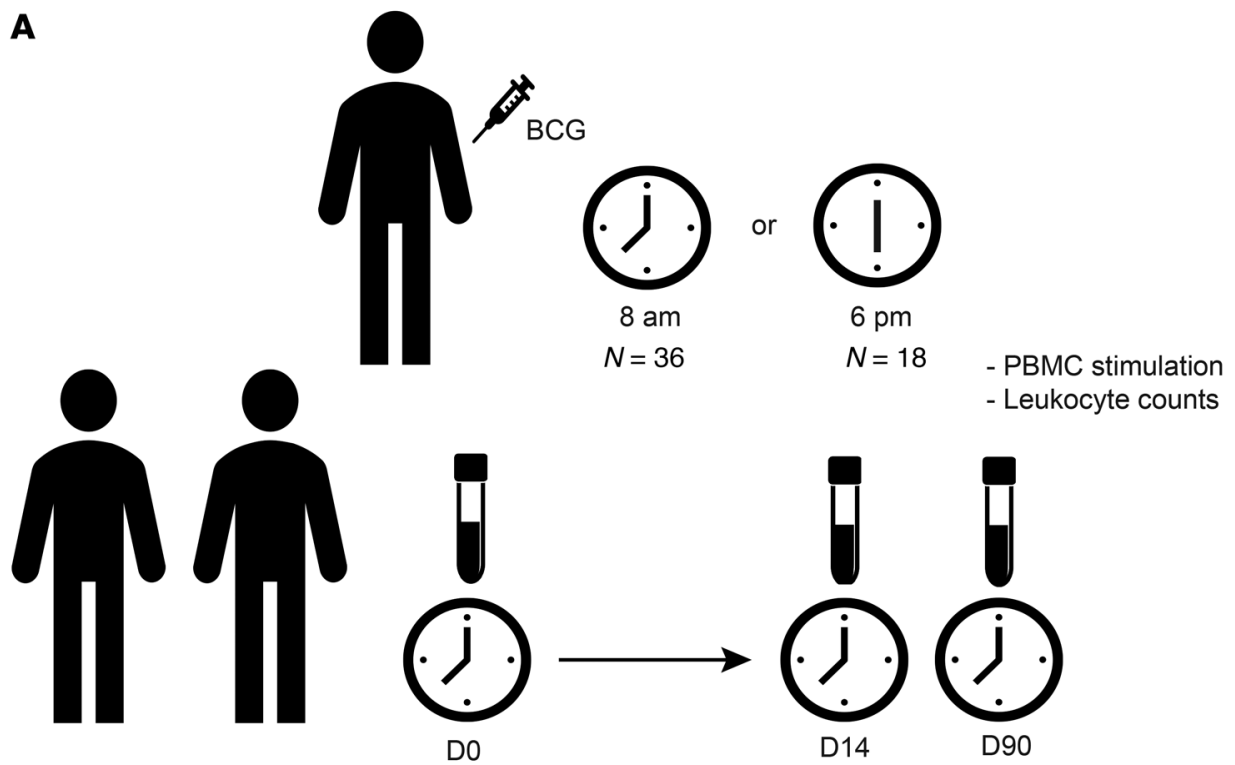

Figure 2. Overview of morningand evening-vaccinated healthy volunteers, including blood counts. (A) Two groups of healthy volunteers were vaccinated with BCG at 2 time points: 18 volunteers between $6 \mathrm{pm}$ and $6: 30 \mathrm{pm}$, while 36 (1:2 ratio) sex- and age-matched controls were vaccinated between 8 am and 9 am. Blood was collected in the morning at baseline, 2 weeks, and 3 months after BCC vaccination. (B) Whole blood complete blood counts and leukocyte differential (neutrophil, lymphocyte, and monocyte counts) of morning-vaccinated individuals and evening-vaccinated individuals. Mean \pm SEM; morning $n=36$, evening $n=18$. Kruskal-Wallis test with Dunn's multiple-comparison test.

B
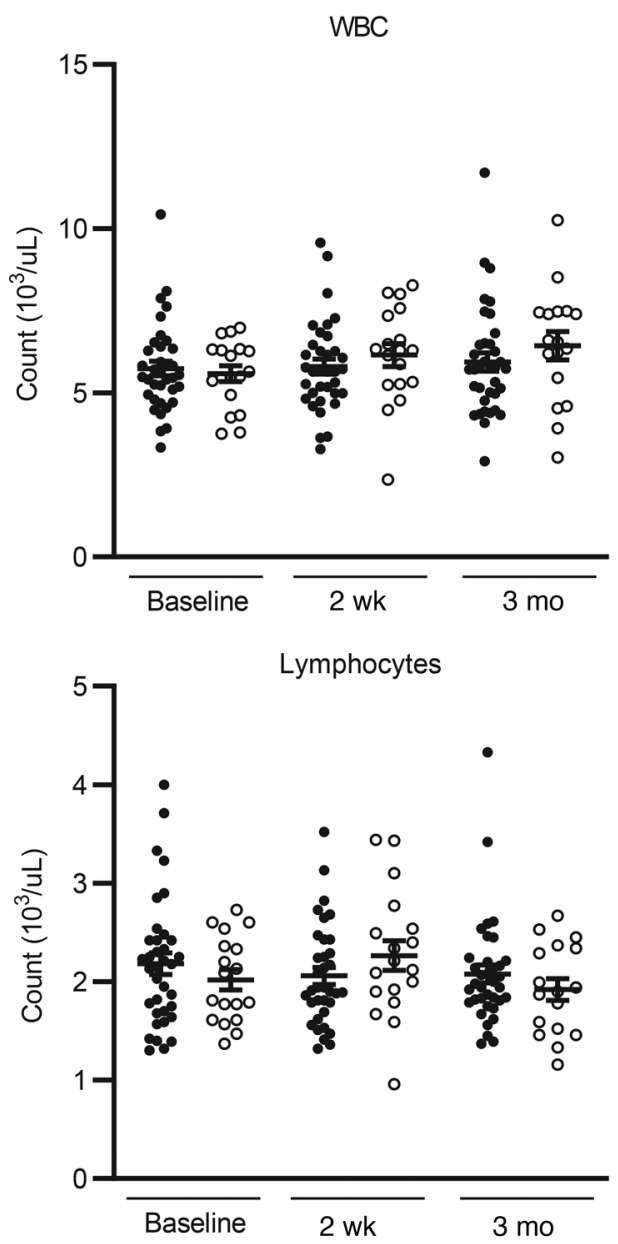

- Morning vaccination o Evening vaccination Neutrophils
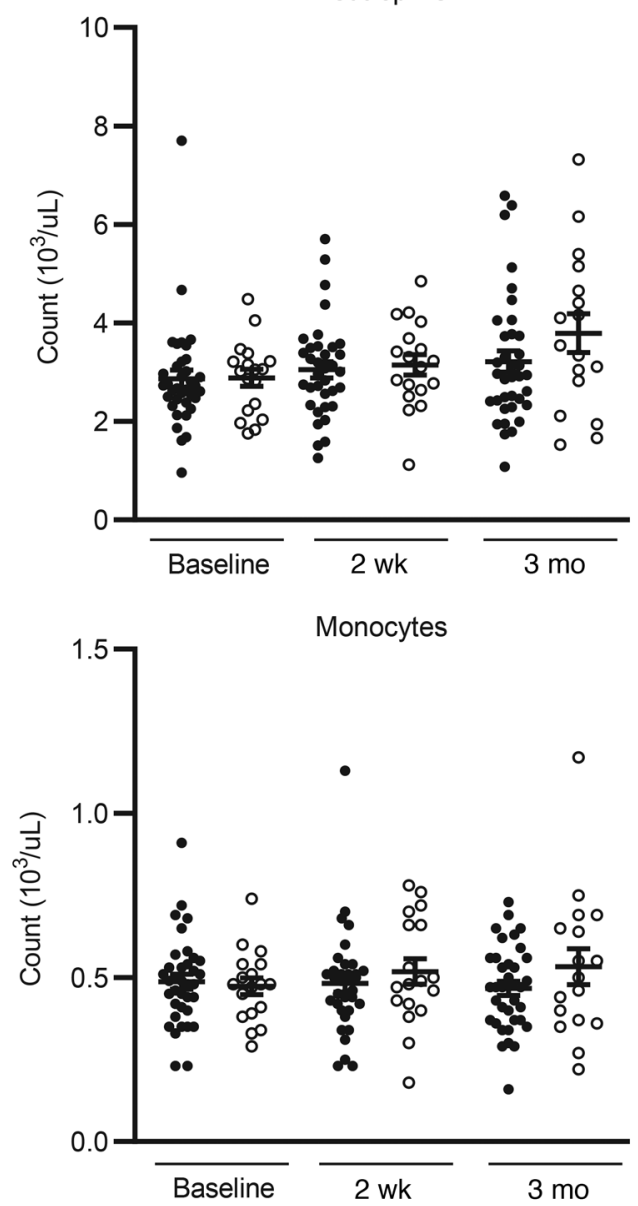

compared with later during the morning (Supplemental Figure 5). We did not find differences in cortisol concentrations between the morning- and evening-vaccinated groups, and no significant correlations were observed between circulating cortisol concen- trations and ex vivo PBMC-derived $S$. aureus-induced IL-1 $\beta$, IL-6, or TNF- $\alpha$ responses, or $M$. tuberculosis-induced IFN- $\gamma$ responses (data not shown). Moreover, addition of morning-derived serum to freshly Percoll-isolated monocytes during 24 hours of prim- 
A

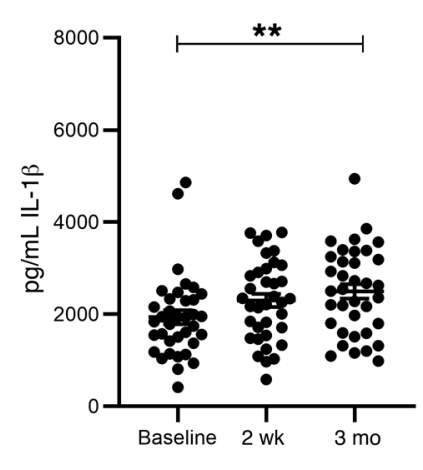

D

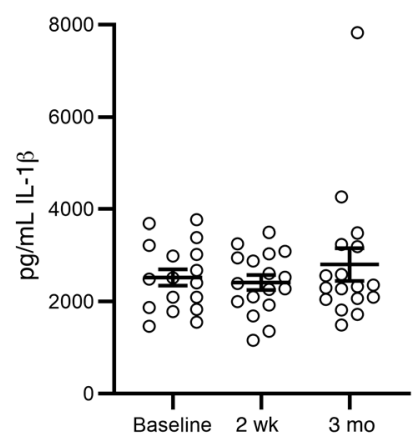

G

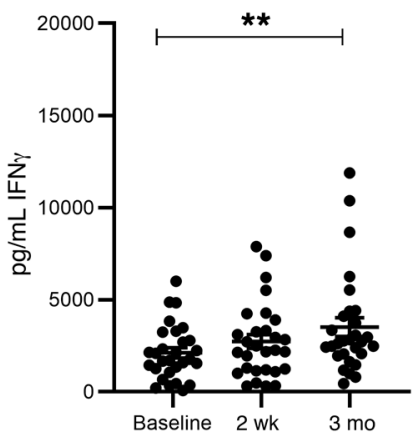

B

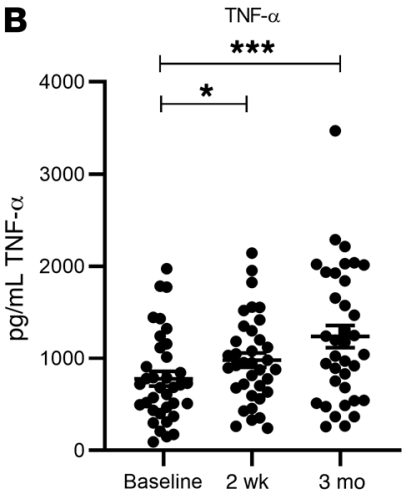

E

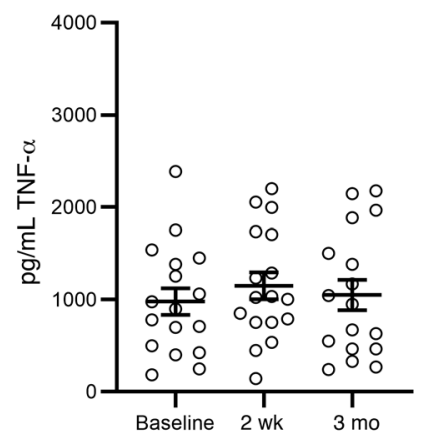

H

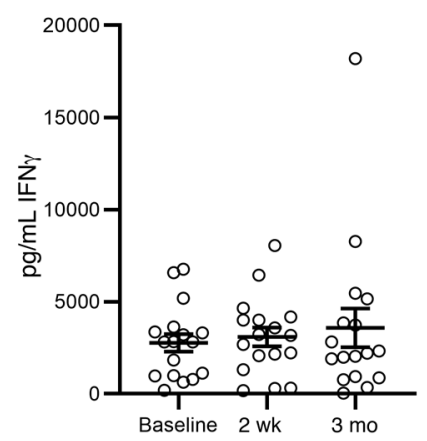

C

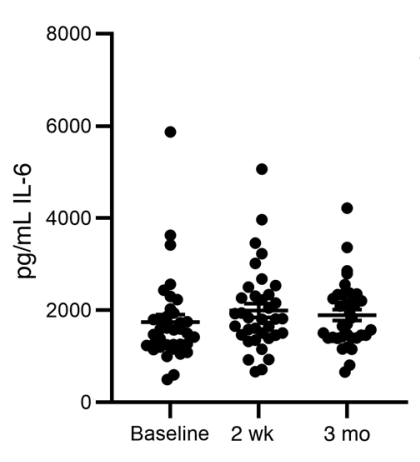

$\mathbf{F}$

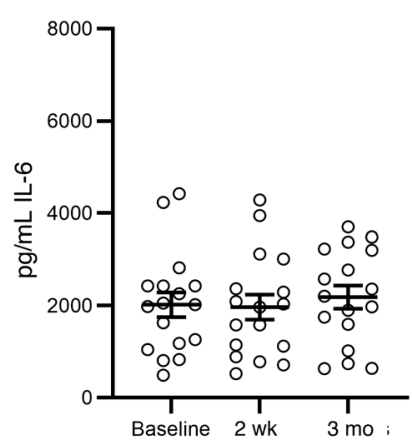

I

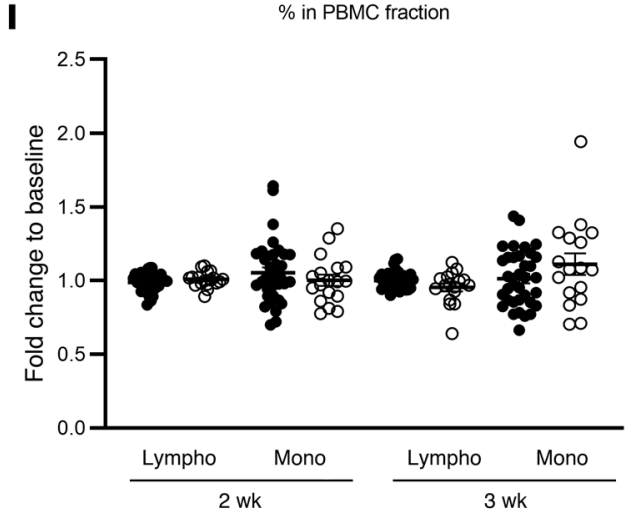

Figure 3. BCG vaccination in the morning elicits a stronger trained immunity phenotype compared with evening vaccination. IL-1 $\beta$, IL-6, and TNF- $\alpha$ production in response to $S$. aureus stimulation 2 weeks and 3 months after BCG vaccination, and production of IFN- $\gamma$ in response to M. tuberculosis (Mtb) stimulation of morning-vaccinated (A-C and $\mathbf{G})$ and evening-vaccinated ( $\mathbf{D}-\mathbf{F}$ and $\mathbf{H}$ ) individuals. Mean $\pm \mathrm{SEM} ; n=36$ morning vaccinated, $n=18$ evening vaccinated ${ }^{* * *} P<0.001,{ }^{* *} P<0.01,{ }^{*} P<0.05$ by Friedman's test with Dunn's multiple-comparison test. (I) Fold changes (compared with baseline) of monocyte and lymphocyte percentages within PBMC fraction. Mean \pm SEM; morning $n=36$, evening $n=18$. Kruskal-Wallis test with Dunn's multiple-comparison test.

ing with BCG had an inhibitory effect on in vitro BCG-induced trained immunity compared with serum collected from volunteers in the evening (Figure 6, A-C), which thus cannot explain the better induction of trained immunity in the morning.

The differential response to the BCG vaccine could also be explained by cell-intrinsic circadian changes. We therefore isolated monocytes from a separate group of healthy volunteers in the morning and in the evening. Indeed, when monocytes were collected and isolated in the morning, they displayed an increased capacity to mount a trained immunity response (higher proinflammatory cytokine production after LPS restimulation) in an experimental in vitro model, compared with cells isolated in the evening
(Figure 6, A, D, and E). No significant differences were found in the production of the antiinflammatory cytokine IL-10 (Figure 6F).

Circadian transcriptional and epigenetic changes in BCG-vaccinated individuals. Furthermore, we studied whether the expression of key clock genes CLOCK and ARNTL (BMAL1) are affected in morning BCG-vaccinated individuals from an independent cohort (20). As seen in Figure 7, CLOCK and ARNTL mRNA expression in monocytes was increased 1 month after BCG vaccination in individuals who were protected against subsequent yellow fever viremia (responders). Additionally, histone 3 lysine 27 acetylation (H3K27ac), an epigenetic modification associated with gene activation, was increased at the CLOCK and ARNTL 
A

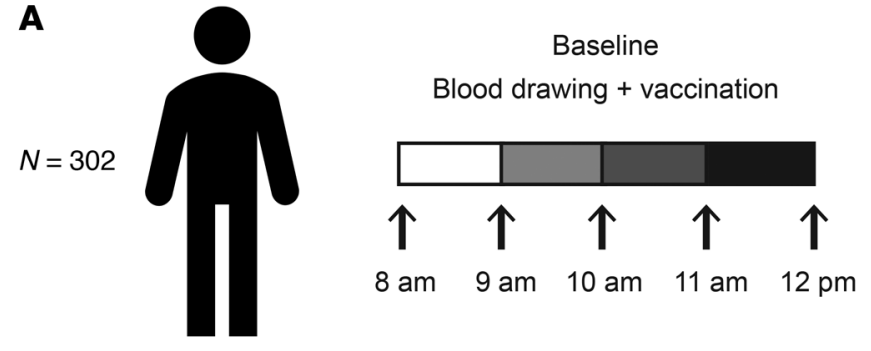

2 wk

Blood drawing

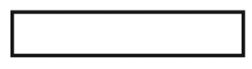

$8 \mathrm{am}-10 \mathrm{am}$
$3 \mathrm{mo}$

Blood drawing
B

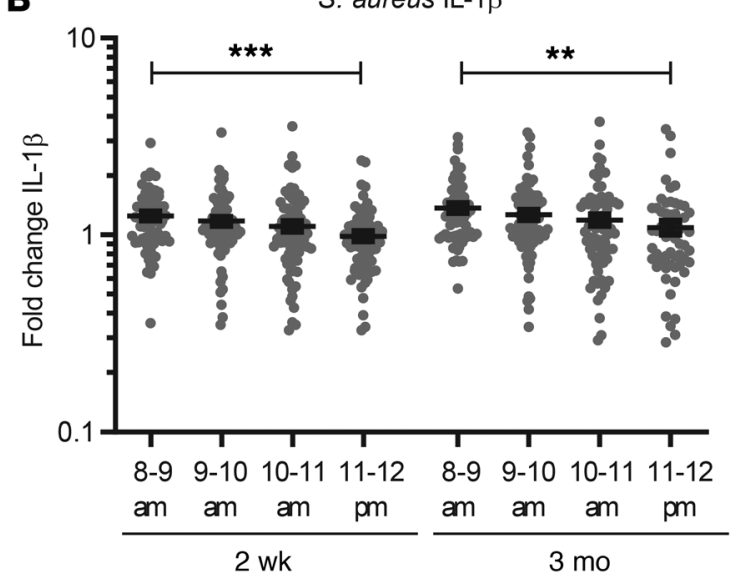

D

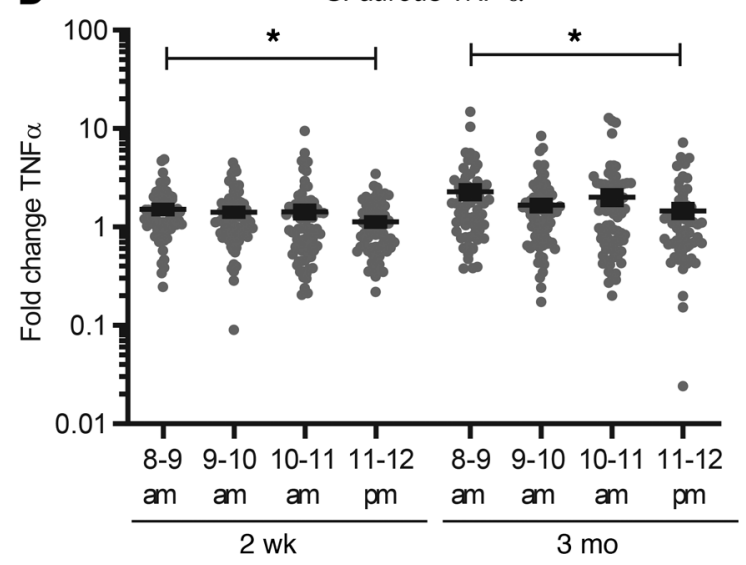

C

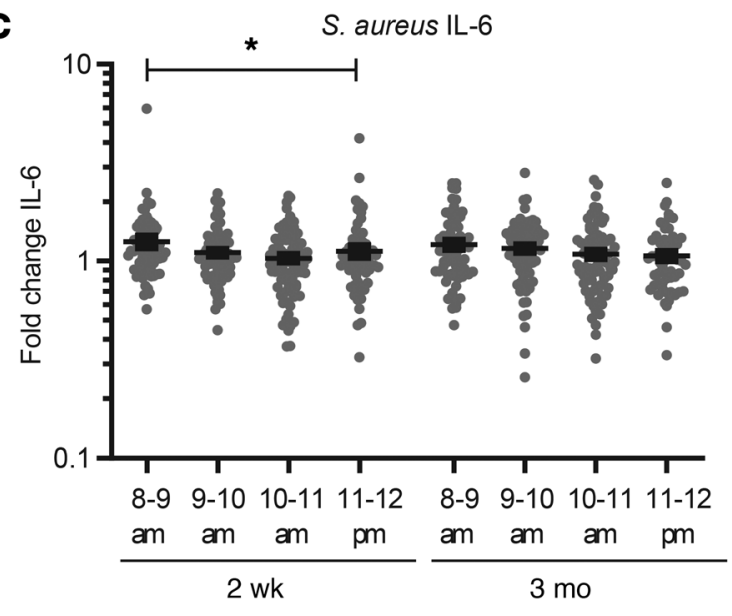

E

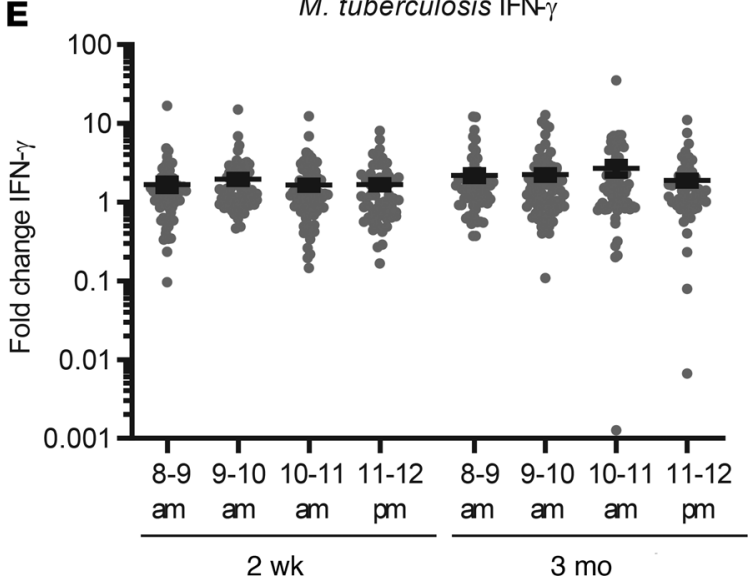

Figure 4. Trained immunity responses induced by BCG vaccination are most profound in the early morning. (A) A total of 302 healthy volunteers were BCG vaccinated between 8 am and 12 pm and blood was collected before, 2 weeks after, and 3 months after BCG vaccination. Fold changes (compared with baseline) 2 weeks and 3 months after BCG vaccination of PBMC-produced IL-1 $\beta$ (B), IL-6 (C), and TNF- $\alpha$ (D) in response to S. aureus stimulation, and IFN- $\gamma$ production in response to $M$. tuberculosis stimulation (E). Mean \pm SEM; $n=68$ vaccinated between 8 am and 9 am, $n=80$ vaccinated between 9 am and $10 \mathrm{am}, n=84$ vaccinated between 10 am and $11 \mathrm{am}, n=66$ vaccinated between 11 am and $12 \mathrm{pm} .{ }^{* * *} P<0.001,{ }^{* *} P<0.01,{ }^{*} P<0.05$ by Kruskal-Wallis test with Dunn's multiple-comparison test.

promoters 1 month after BCG vaccination. Since cells were available only from a limited number of individuals, caution in the interpretation of the results is required.

Since epigenetic rewiring is one of the hallmarks of trained immunity, we assessed the accessibility of chromatin in eveningversus morning-vaccinated individuals by assay for transposaseaccessible chromatin using sequencing (ATAC-seq) (see Supplemental Figure 6 and Supplemental Table 2 for quality statistics). Figure 8A shows differences in chromatin accessibility 3 months after BCG vaccination for evening- compared with morning- vaccinated individuals (see also Supplemental Table 3). Less extensive differences were seen 2 weeks after vaccination (Supplemental Figure 7), which suggests that the epigenetic changes induced by BCG need a relatively long time to become apparent. Individuals vaccinated in the morning had more accessible chromatin in genes of PBMCs important for the mTOR pathway, which is crucial for the induction of trained immunity. The evening-vaccinated individuals showed increased chromatin accessibility in PBMCs for TNF signaling and NOD-like receptor (NLR) signaling (Figure 8B and Supplemental Table 4), which do not explain the higher TNF production 

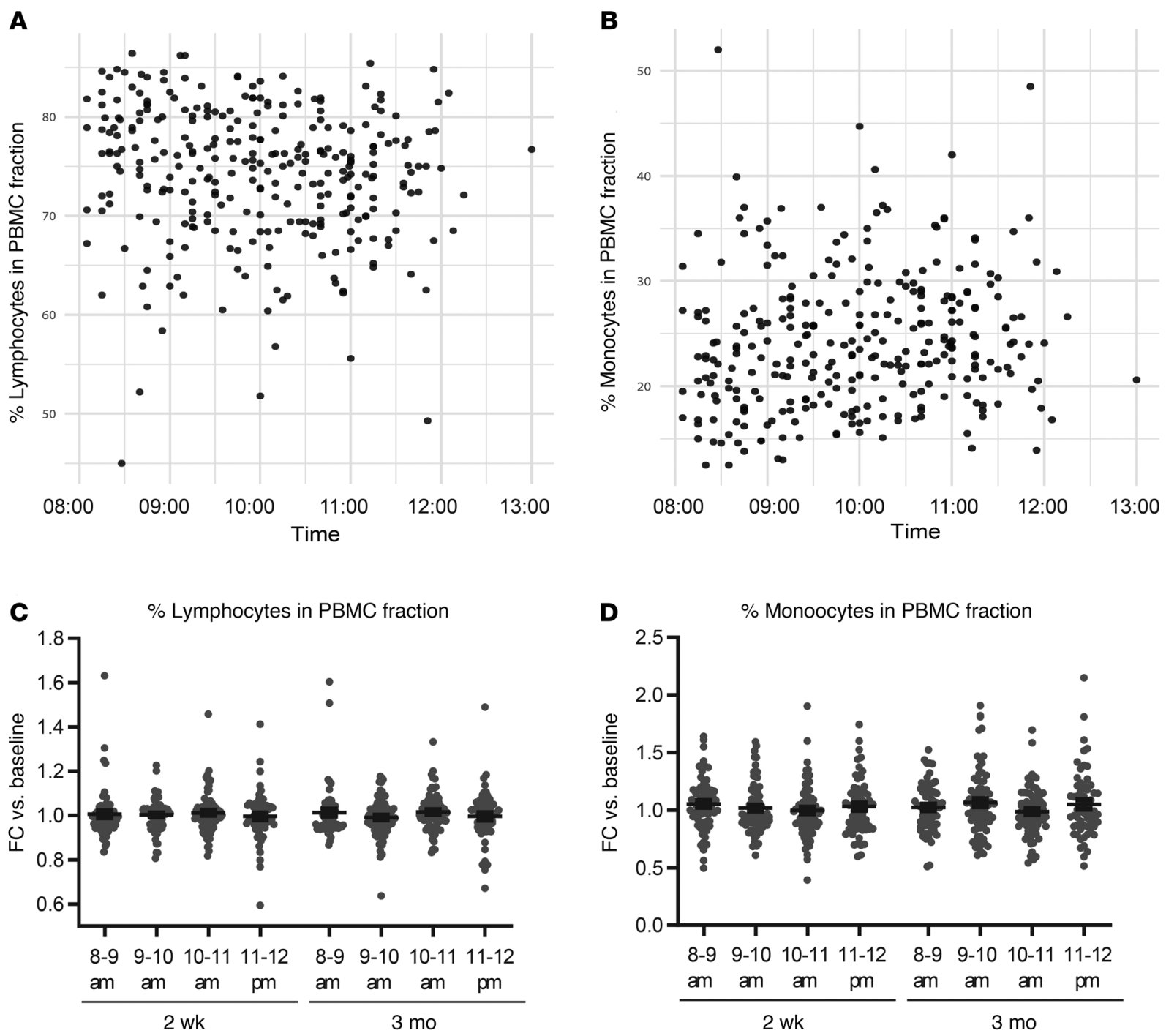

Figure 5. Percentages of monocytes and lymphocytes in PBMC fraction. (A and B) Spearman's correlation plots of lymphocyte percentages $(P=0.004)$ (A) and monocyte percentages $(P=0.002)(B)$ within the PBMC fraction against time of blood collection at baseline visit. (C and $\mathbf{D})$ Comparisons of fold changes (FC) in lymphocyte (C) and monocyte percentages (D) between morning-vaccinated subgroups. Mean \pm SEM; $n=68$ vaccinated between 8 am and $9 \mathrm{am}, n=80$ vaccinated between 9 am and $10 \mathrm{am}, n=84$ vaccinated between 10 am and $11 \mathrm{am}, n=66$ vaccinated between 11 am and 12 pm. Kruskal-Wallis test with Dunn's multiple-comparison test.

capacity in the morning. This suggests that more molecular mechanisms should be activated and involved in BCG-induced trained immunity. Analysis of transcription factor (TF) binding profiles from JASPAR also showed enrichment of TFs involved in mTOR signaling for morning-vaccinated individuals (Figure 8C and Supplemental Table 5). TF binding profiles from CODEX, which focusesonhematopoieticcellines, additionally showsenrichmentof TFs associated with active histone marks and with removal of repressive histone marks in morning-vaccinated individuals (Figure 8D and Supplemental Table 5).

\section{Discussion}

An increasing amount of evidence shows that BCG vaccination elicits a nonspecific innate immune memory phenotype (15-17, $20,23)$, likely contributing toward decreased overall morbidity and mortality after neonatal BCG vaccination (7-10). However, immunological effects after BCG vaccination are highly variable in both children and adults $(7,20,23,36)$, and understanding sources of this variation would be of great importance for attempts to improve vaccination strategies. Circadian rhythms play an important role in modulation of immune function $(27,37,38)$, and we hypothesized that the time of BCG administration might be an important factor to explain the variability of the immunological effects and protective efficacy of the vaccine. In line with this hypothesis, the present study demonstrates that timing of BCG administration is an important factor influencing both nonspecific induction of trained immunity as well as M. tuberculosis-specific responses after vaccination. Early morning vaccination resulted in superior cytokine production capacity upon ex vivo restimulation with related (M. tuberculosis) and unrelated pathogens (S. aureus) compared with evening vaccination. Strikingly, both the nonspecific trained innate immunity as well as M. tuberculosis-specific 
A

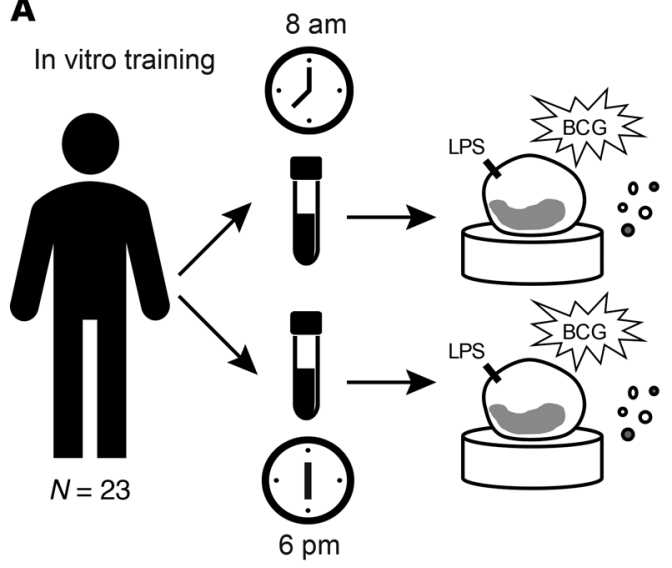

B

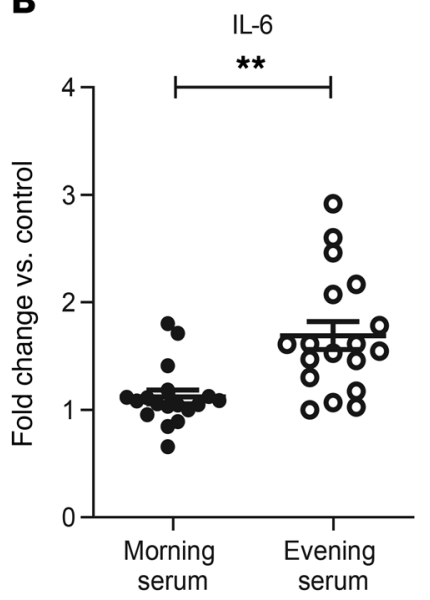

C

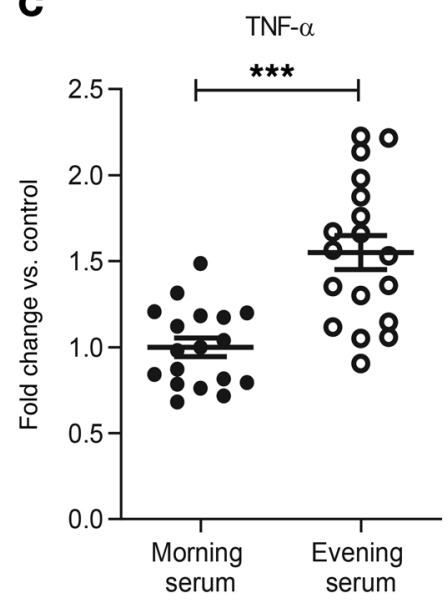

D

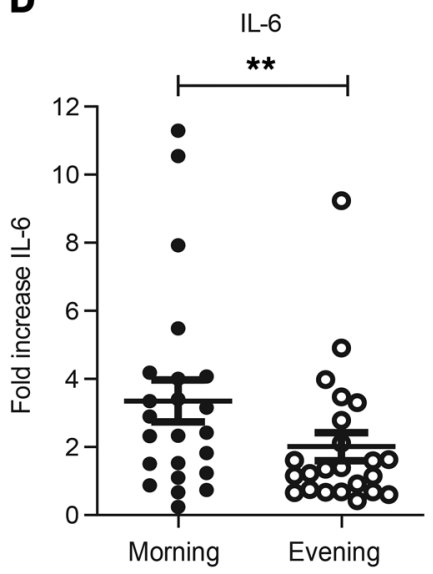

$\mathbf{E}$

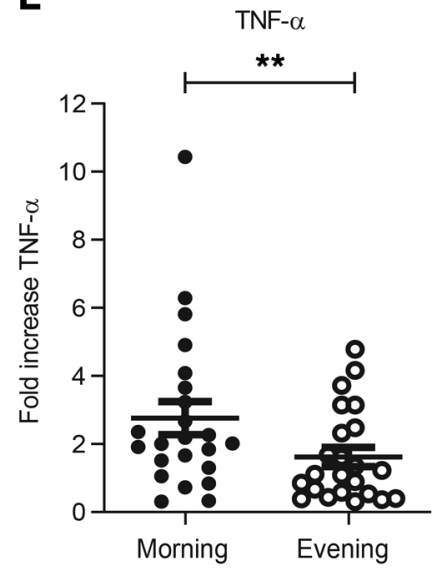

$\mathbf{F}$

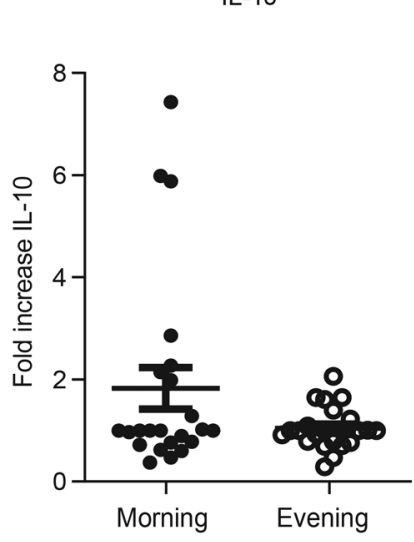

Figure 6. Trained immunity responses in vitro are affected by time of the day. (A) Blood was collected from healthy volunteers for isolation of serum and Percoll purification of monocytes during the morning $(8 \mathrm{am})$ and evening $(6 \mathrm{pm})$ on the same day. (B and $\mathbf{C})$ Fold changes (compared with medium-primed, LPS-restimulated conditions) in IL-6 (B) and TNF- $\alpha$ (C) production of BCG-primed monocytes supplemented with morning-derived serum versus eveningderived serum. (D-F) Fold changes (relative to medium-primed, LPS-restimulated conditions) in IL-6 (D), TNF- $\alpha$ (E), and IL-10 (F) production after LPS restimulation of BCG-trained monocytes derived after morning blood donation versus evening blood donation. Mean \pm SEM; $n=18$ morning evening serum, $n=23$ morning evening monocytes. ${ }^{* * *} P<0.001,{ }^{* *} P<0.01$ by Wilcoxon's matched-pairs signed-rank test.

adaptive responses were practically absent in the volunteers vaccinated in the evening. Furthermore, the earlier volunteers were vaccinated in the morning, the stronger the trained immunity phenotype in terms of cytokine production.

Our findings are in line with previous studies showing enhanced immunogenicity after influenza and hepatitis A vaccination in the morning compared with the evening $(30,31)$. The findings from our study confirm previous observations in mice showing an effect of timing of BCG administration on immune function. For example, neutrophil migration in mice implemented with BCG-impregnated cell traps displayed a circadian rhythm $(39,40)$. In an experimental BCG inflammation mouse model, a diurnal inflammatory response was noticed at the site of inoculation 30 days after BCG administration (41). In another study, circadian oscillations were observed in nonspecific protection of BCG-treated mice at different times during the day and subsequent Ehrlich ascites carcinoma challenge (42). Interestingly, the magnitude of infection by the protozoan Leishmania has been shown to be modulated by a circadian clock in immune cells (43), and one of its causative species, Leishmania braziliensis, is a potent inducer of in vitro-trained immunity (44). Trained immunity responses induced by $S$. aureus and specific memory responses to $M$. tuberculosis upon BCG vaccination are differently affected by the circadian rhythm. Previous studies showed that different types of viruses display distinct interactions with core clock proteins (45). Similar differences might explain the different contribution of the circadian rhythm observed in this study to cytokine production upon $S$. aureus versus $M$. tuberculosis stimulation, but this needs to be investigated in future studies.

Chromatin accessibility showed differences between eveningand morning-vaccinated volunteers 3 months after vaccination. Importantly, these differences were not present after 2 weeks, indicating a relatively long period needed by BCG vaccination to induce trained immunity. Chromatin that was more accessible for morning-vaccinated volunteers was enriched for genes of the mTOR pathway, which is crucial for trained immunity (17). This is in line with the observed more-pronounced cytokine production in the morning-vaccinated individuals. Unexpectedly, several genes 
CLOCK

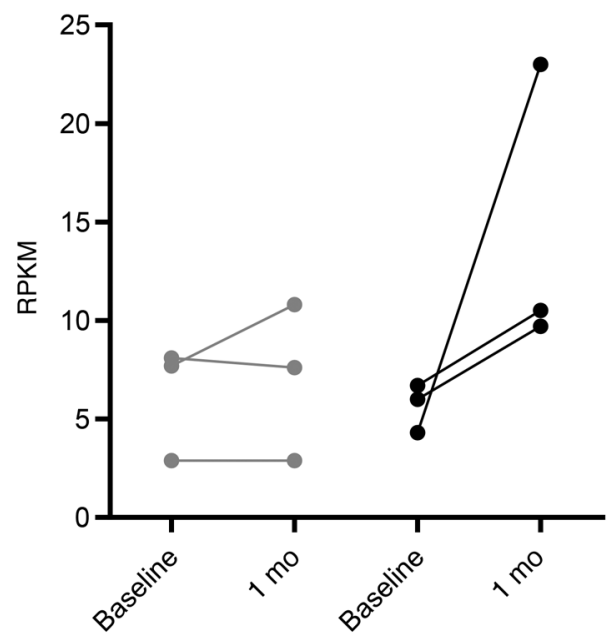

ARNTL

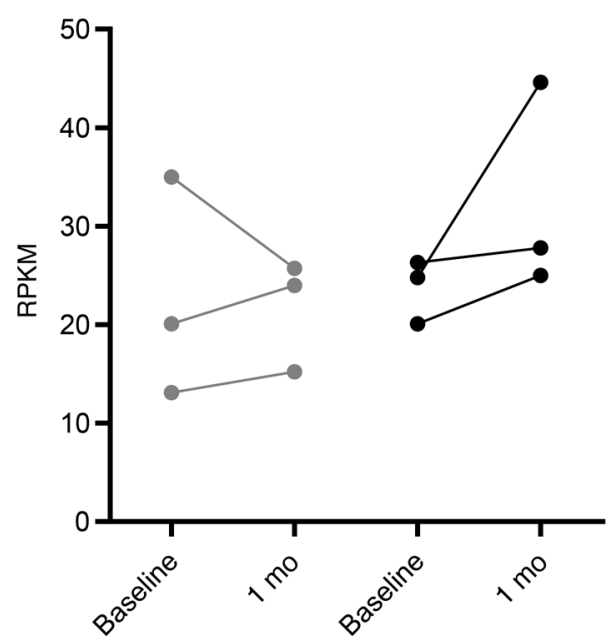

CLOCK
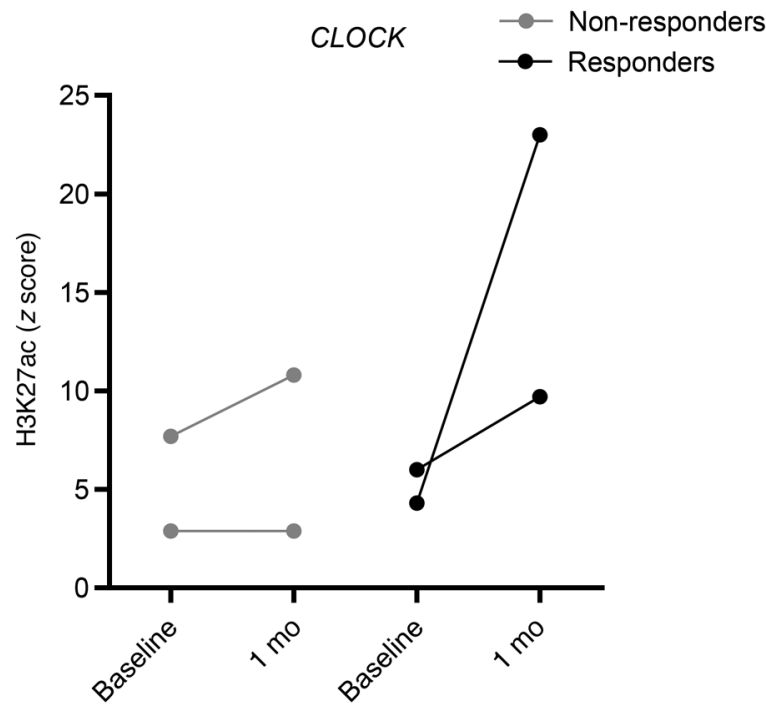

ARNTL

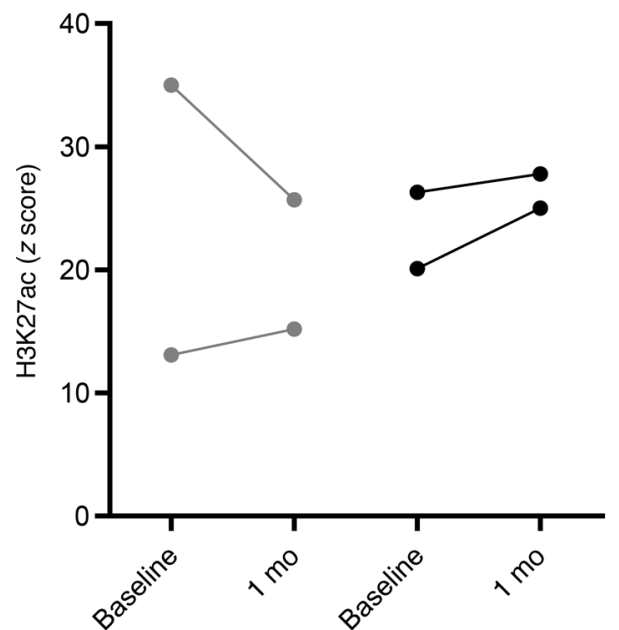

Figure 7. mRNA expression of CLOCK and ARNTL in monocytes from individuals before and 1 month after BCG vaccination. Individuals were divided into responders, which were protected from subsequent yellow fever viremia (maximum yellow fever viremia $\mathrm{CT}>36[n=3]$ ), and nonresponders $(\mathrm{CT}<36$ [ $n=$ 3]). H3K27ac levels at gene promoters (Z score) of CLOCK and ARNTL before and 1 month after BCG vaccination in monocytes ( $n=2$, both groups).

of the TNF and NLR signaling pathways had decreased accessibility in the PBMCs of morning-vaccinated individuals, which argues that more molecular mechanisms are at play. In addition, enrichment was observed of TFs associated with mTOR signaling for the individuals with BCG administration in the morning: specificity protein 1 (SP1) $(46,47)$, TF AP-2 $\alpha$ (TFAP2A) (47), and Krüppel-like factor 4 (KLF4) $(48,49)$.

Recently we have shown that the longevity of BCG-induced trained immunity in humans is explained by an imprint on hematopoietic stem and progenitor cells (HSPCs), as demonstrated by a bias toward myelopoiesis and upregulated myeloid function at the transcriptional level of HSPCs (19). The results of the current study suggest that the impact of BCG on bone marrow progenitors depends on the time of day when the vaccine was administered. There are several hypotheses predicting how the priming of bone marrow precursors could be under the influence of a circadian rhythm. First, a molecular intrinsic clock within monocytes and their progenitors could contribute toward the observed effects. This hypothesis is supported by the experiments performed here showing that monocytes isolated from healthy volunteers in the morning mount a stronger trained immunity phenotype if exposed ex vivo to BCG. Similarly, we demonstrated that monocyte production of IL-1 $\beta$ was partially influenced by the time of the day. These data could explain our findings that BCG administration time influences trained immunity in vivo, as well as that the time of blood drawing determines the efficacy in induction of in vitro-trained immunity. Regulators of cytokine secretion and pathogen recognition receptors are examples of genes that have been shown to oscillate at a transcriptional level in a circadian manner in macrophages (26). Here we show that the key clock genes, CLOCK and ARNTL, are more strongly expressed in monocytes 1 month after BCG vaccination, accompanied by increased H3K27ac at the gene promoters (when blood was taken at the same time point in the morning). Multiple studies have 
A

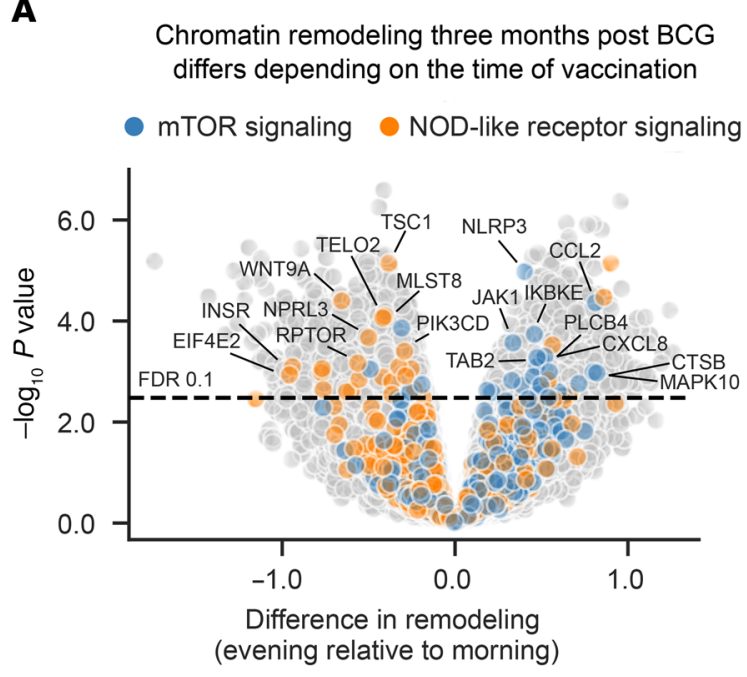

C

JASPAR TFBSs enrichment of regions for which remodeling differs depending on the time of vaccination

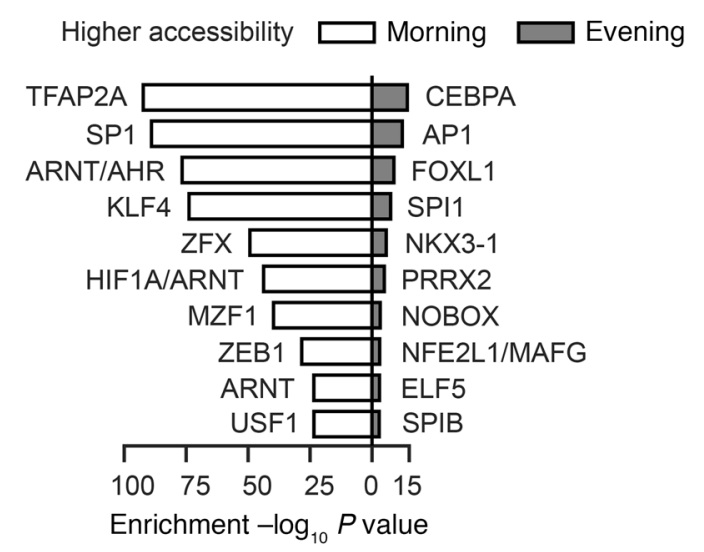

B

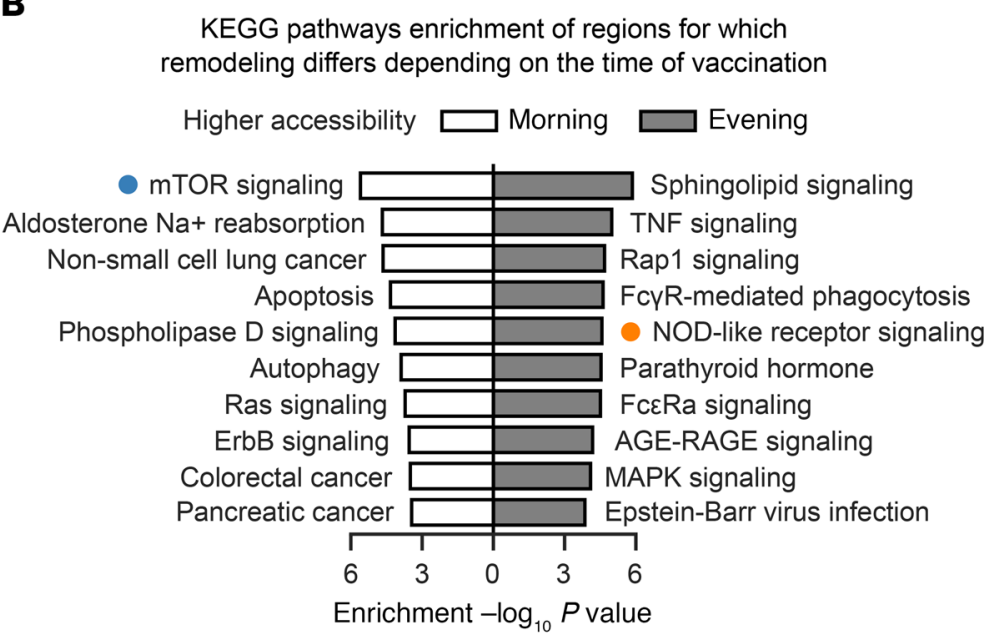

D

CODEX TFBSs enrichment of regions for which remodeling differs depending on the time of vaccination

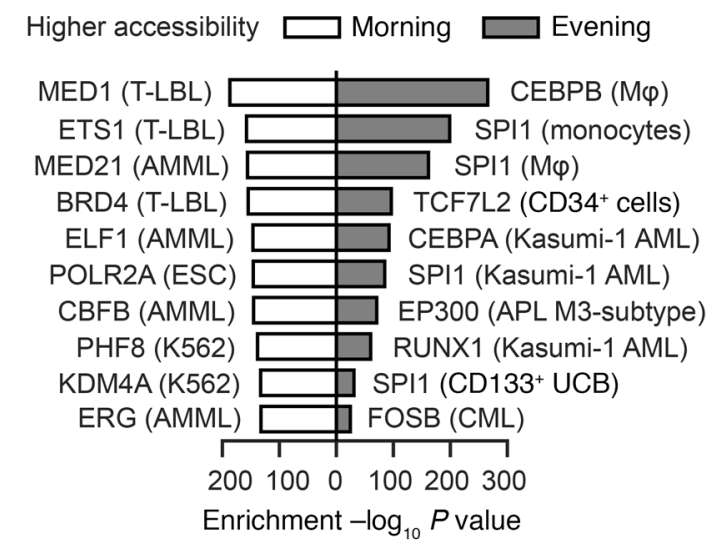

Figure 8. The effect of BCG vaccination on epigenome remodeling in PBMCs differs based on the time of vaccination. Differential chromatin accessibility (DA) analysis of an interaction effect between BCG training (3 months after BCG compared with baseline) and time of vaccination (evening compared with morning). (A) Open chromatin regions were assigned to genes based on proximity. (B) KEGG pathways enrichment of regions for which remodeling 3 months after BCG differs based on the time of vaccination (showing top 10 pathways for each direction, all displayed pathways passed FDR of 0.02 ). (C and D) JASPAR and CODEX transcription factor binding sites (TFBSs) enrichment of regions for which remodeling 3 months after BCC differs based on the time of vaccination (showing top 10 TFs for each direction, all displayed TFs passed FDR of 0.005). $n=36$ morning vaccinated, $n=18$ evening vaccinated; DA was performed with LIMMA, which computes $P$ values with a moderated $t$ test; enrichment analysis was performed with a Fisher's exact test; BenjaminiHochberg procedure was used to control the FDR.

shown that the time of day of the infection (bacterial, viral, and parasitic) affects the outcome of the infection (50). Previously, it has been shown in mice that low expression of BMAL1 led to increased herpes, influenza (51), and respiratory syncytial virus infection (52). One could hypothesize that increased CLOCK and BMAL1 expression upon BCG vaccination contributes to the observed effect: better protection against heterologous infections upon BCG vaccination.

Second, an intrinsic neutrophil timer might be involved in the time-dependent induction of trained immunity by BCG in vivo. Interestingly, mouse studies have shown that neutrophils, and not dendritic cells or macrophages, transfer live BCG bacilli to draining lymph nodes (53). In an intradermal modified vaccinia Ankara (MVA) vaccination mouse model, CD8 memory responses were surprisingly elicited in the bone marrow compartment, mediated by neutrophils trafficking MVA from the dermis to the bone marrow (54). Recently, a neutrophil timer was discovered that coordinates immune defense (25). One of the core clock proteins, BMAL1, as well as the chemokine CXCR2, have been shown to regulate tissue migration and neutrophil clearance (25). However, it remains to be proven by future studies that neutrophils play a role in the circadian effects of BCG vaccination.

Third, it has been shown in mice that hematopoietic stem cell release is regulated by circadian oscillations, and that HSPCs and leukocytes circulate between bone marrow and peripheral blood following circadian oscillations $(55,56)$. These circadian oscillations may influence the trained immunity phenotype of the myeloid progeny. Of note, HSPCs are known to express pathogen recognition receptors as well, which are known to oscillate on a transcriptional level in monocytes in a circadian 
manner $(57,58)$. Another hypothesis is that the circadian rhythm induction of trained immunity is mediated by IL-1 $\beta$, one of the key cytokines in BCG-induced trained immunity in vivo (20), the production of which has lately been shown to be under the control of the circadian clock protein BMAL1 (59). Finally, a soluble rather than cellular substrate of circadian effects may be hypothesized, since multiple hormones and lipids display diurnal variations in concentration in the circulation (35). Among them, one of the prime candidates for a modulatory role during the circadian rhythm is cortisol. Cortisol has long been known to display a circadian rhythm in concentrations in the blood (35), and its immunomodulatory effects are also well documented (60). However, a role for cortisol is unlikely as it is a known immunosuppressive factor, yet its highest circulating concentration is in the morning, when induction of trained immunity is most effective. Moreover, incubation of monocytes with serum collected in the morning did not result in an increased induction of trained immunity in an in vitro model, arguing that a soluble factor is unlikely to be responsible for the circadian effects of BCG vaccination. Nevertheless, we do not exclude possible involvement of circulating factors, such as insulin, hormones, or dietary components, which could dampen BCG-induced trained immunity in the morning. However, this effect might be masked by cell-intrinsic circadian oscillations.

In conclusion, in the present study we demonstrate the importance of timing of BCG vaccine administration, with a preference for vaccination in the early morning to induce both trained immunity and M. tuberculosis-specific adaptive responses. This effect was most likely mediated by an intrinsic circadian clock of innate immune cells, rather than soluble factors in the circulation that display circadian rhythms. Future studies should test whether optimized timing of BCG administration results in increased protection after challenge with unrelated pathogens in vivo. Moreover, deciphering the mechanism behind the influence of time of vaccination on induction of trained immunity and M. tuberculosis-specific responses could lead to possible targets to increase BCG efficacy. Since this study focused exclusively on individuals of Western European ancestry, our findings should be validated in cohorts with a different environmental setting and genetic background. Finally, time of administration of novel and already existing vaccines should be taken into account when testing specific and nonspecific vaccine efficacy. These findings suggest that administering partially effective vaccines like BCG in the morning, in contrast to after school (showing 50\% efficacy against sustained infection after adolescent revaccination; ref. 61), might increase immune training and thus vaccine efficacy. This possible effect remains speculative but within the realm of possibility, and it should be investigated in future studies.

\section{Methods}

Experimental design. The effect of timing of BCG administration on the induction of trained immunity in vivo was studied as a nested substudy within the 300BCG cohort. In the 300BCG study, 321 healthy (male and female) adult volunteers of Western European ancestry were included from April 2017 until June 2018. Exclusion criteria were use of systemic medication other than oral contraceptives and acetaminophen, use of antibiotics 3 months before inclusion, previ- ous BCG vaccination, history of TB, any febrile illness 4 weeks before participation, vaccination 3 months before participation, and a medical history of immunodeficiency. Healthy volunteers were recruited using flyers and advertisements in Nijmegen, and received compensation. At the Radboud University Medical Center, blood was collected, followed by administration of a standard dose of $0.1 \mathrm{~mL} \mathrm{BCG}$ Bulgaria (InterVax) intradermally in the left upper arm by a medical doctor. Additionally, blood was collected in the morning 2 weeks as well as 3 months after vaccination.

Volunteers participating in the 300BCG trial were vaccinated in the morning (between 8 am and $12 \mathrm{pm}$ ). In order to be able to study differences between morning- and evening-vaccinated individuals, 18 (7 male and 11 female) volunteers were asked to be vaccinated between $6 \mathrm{pm}$ and $6: 30 \mathrm{pm}$. All participants, including the volunteers that received their BCG vaccination in the evening, donated blood for immunological assessments in the morning between 8 am and 12 am, and no significant changes in time of blood drawing before versus after vaccination were seen within the evening- and morning-vaccinated groups. For the analysis, each volunteer vaccinated in the evening was age and sex matched with 2 participants vaccinated in the morning between 8 am and 9 am, resulting in 36 matched controls. A schematic overview of the nested morning-evening study can be found in Figure 2A. The 18 evening-vaccinated volunteers were included in morning-evening substudy analyses but excluded from the main cohort. One volunteer belonging to the main cohort was excluded from analysis because the vaccination time was not registered, resulting in a cohort with 302 morning-vaccinated volunteers (see Figure 1).

RNA expression of clock genes was measured in healthy individuals vaccinated with BCG from the BCG-Yellow Fever study, and was performed as described previously (GSE104149; ref. 20). The study was approved by the Arnhem-Nijmegen Medical Ethical Committee (NL50160.092.24).

PBMC isolation and stimulation. PBMCs were isolated from EDTA whole blood with Ficoll-Paque (GE Healthcare) density gradient separation. PBMCs were washed twice with phosphate-buffered saline (PBS) and counted with a Sysmex hematology analyzer (XN-450). Cells were suspended in Dutch-modified Roswell Park Memorial Institute (RPMI) 1640 medium (Invitrogen), supplemented with 50 $\mu \mathrm{g} / \mathrm{mL}$ gentamicin, $2 \mathrm{mM}$ Glutamax (GIBCO) and $1 \mathrm{mM}$ pyruvate (GIBCO). PBMCs $\left(5 \times 10^{5}\right)$ were cultured in a final volume of $200 \mu \mathrm{L} /$ well in round-bottom 96-well plates (Greiner) and stimulated with RPMI (medium control), heat-killed M. tuberculosis HR37v (5 $\mu \mathrm{g} / \mathrm{mL})$, or heat-killed S. aureus $\left(1 \times 10^{6} \mathrm{CFU} / \mathrm{mL}\right.$, clinical isolate $)$ as a nonspecific stimulus and incubated at $37^{\circ} \mathrm{C}$. After 24 hours and 7 days, supernatants were collected and stored at $-20^{\circ} \mathrm{C}$ until analysis. Cytokines (IL-1 $\beta$, IL-6, and TNF- $\alpha$ ) were determined with ELISA (R\&D Systems) in 24-hour supernatants and IFN- $\gamma$ in 7-day supernatants with Luminex (ProcartaPlex, Thermo Fisher Scientific), according to the manufacturers' protocols. To minimize batch effects during measurements, samples were sorted per stimulus. All time points belonging to one volunteer were measured on the same plate.

In vitro training experiments. In vitro training experiments were performed according to the previously described experimental in vitro-trained immunity model (22). Healthy volunteers donated blood at 8 am (fasting) and $6 \mathrm{pm}$ (fasting from $1 \mathrm{pm}$ ) on the same day. PBMCs were isolated from EDTA whole blood with Ficoll-Paque density gradient separation. Percoll (MilliporeSigma) isolation of 
monocytes was performed according to the previously described protocol (22). Cells were suspended in Dutch-modified RPMI 1640 medium, supplemented with $50 \mu \mathrm{g} / \mathrm{mL}$ gentamicin, $2 \mathrm{mM}$ Glutamax, and $1 \mathrm{mM}$ pyruvate and counted with a Sysmex hematology analyzer $(\mathrm{XN}-450)$. As an additional purification step, $1 \times 10^{5}$ Percoll-isolated monocytes were plated on polystyrene flat-bottom plates (Corning) and incubated for 1 hour at $37^{\circ} \mathrm{C}$, after which nonadherent cells were washed away with warm PBS. Adherent monocytes were primed with either RPMI 1640 (negative control) or BCG Bulgaria $(5 \mu \mathrm{g} / \mathrm{mL})$ for 24 hours in the presence of $10 \%$ pooled human serum. Cells were washed after 24 hours, and fresh medium (RPMI 1640 supplemented with $10 \%$ pooled human serum) was added. Medium was refreshed once after 3 days. After 6 days, cells were restimulated with RPMI 1640 (negative control) or LPS (10 ng/ $\mathrm{mL})$. IL-6, TNF- $\alpha$, and IL-10 concentrations were determined with ELISA in harvested culture supernatants according to the manufacturer's instructions. All conditions belonging to one volunteer were measured on the same plate.

Chromatin accessibility mapping by ATAC-seq. ATAC-seq was performed as previously described $(62,63)$, with minor adaptations. In each experiment, 50,000 PBMCs were collected at $300 \mathrm{~g}$ and $4^{\circ} \mathrm{C}$ for 5 minutes. After centrifugation, the pellet was carefully resuspended in the transposase reaction mix $(12.5 \mu \mathrm{L} 2 \times$ TD buffer, $2 \mu \mathrm{L}$ TDE1 [Illumina], $10.25 \mu \mathrm{L}$ nuclease-free water, and $0.25 \mu \mathrm{L} 1 \%$ digitonin [Promega]) for 30 minutes at $37^{\circ} \mathrm{C}$. Following DNA purification with the MinElute kit eluting in $11 \mu \mathrm{L}, 1 \mu \mathrm{L}$ of eluted DNA was used in a qPCR reaction to estimate the optimum number of amplification cycles. The remaining $10 \mu \mathrm{L}$ of each library was amplified for the number of cycles corresponding to the Cq value (the cycle number at which fluorescence has increased above background levels) from the qPCR. Library amplification was followed by SPRI (Beckman Coulter) size selection to exclude fragments larger than $1,200 \mathrm{bp}$. DNA concentration was measured with a Qubit fluorometer (Life Technologies). Library amplification was performed using custom Nextera primers. Libraries were sequenced by the Biomedical Sequencing Facility at CeMM using the Illumina HiSeq 3000/4000 platform and the 50-bp single-end configuration. Chromatin accessibility mapping by ATACseq was done in 2 biologically independent experiments. Sequencing statistics are provided in Supplemental Table 2.

Chromatin accessibility data processing. ATAC-seq reads were trimmed using Skewer (64) and aligned to the GRCh38 assembly of the human genome using Bowtie 2 (65) with the "-very-sensitive" parameter. Duplicate reads were removed using the sambamba (66) "markdup" command and reads with mapping quality $\geq 30$ and alignment to the genome were kept. Peak calling was performed with MACS2 (67) using the "-nomodel” and "-extsize 147" parameters, and peaks overlapping blacklisted features as defined by the ENCODE project (68) were discarded. Transposase-cutting loci were generated from the filtered BAM files, taking into account the transposase bias in a strand-specific way.

We calculated the following sample quality statistics. The fraction of reads in peaks (FRIP) was calculated as the fraction of transposase cutting events overlapping identified peaks. Similarly, we quantified the fraction of transposase cutting events overlapping all regions defined in the Ensembl Regulatory Build (Oracle_FRIP) (69) and the fraction of peaks overlapping the promoters (Promoter FRIP). We calculated the transcription start site (TSS) enrichment plot as the histogram of transposase cutting events in the peaks around all TSSs $( \pm 1,000 \mathrm{bp}$ ), normalized by the average tail value (calculated as the average coverage on the last $100 \mathrm{bp}$ of both the left and right tails of the histogram). The TSS_enrichment value was defined as the maximum value of the enrichment plot.

Upon examining the sample quality statistics, we selected samples with at least 100,000 sequenced reads and 5,000 called peaks for further analysis. Next, peak lists were aggregated to a consensus peak list by merging called peak summits extended on both sides by $250 \mathrm{bp}$ across all samples that passed the quality control. We quantified the accessibility of each consensus peak in each sample by counting the number of transposition events that overlapped the peak.

The peaks were annotated using UROPA (70) with features defined based on the GENCODE v31 reference annotation (69) as follows: TSS if the peak was within \pm 100 bp from the TSS, TSS proximal if the peak was within 1,000 bp upstream or 500 bp downstream from the TSS, gene_body if the peak overlapped a gene, distal if the peak was within 100,000 bp from the TSS, or intergenic otherwise. For each peak only the closest GENCODE feature was considered and the annotations took precedence in the following order: TSS, TSS proximal, gene_body, distal, and intergenic.

Differential chromatin accessibility analysis. To identify open chromatin regions that respond differently to training induced by BCG conditioned on the time of vaccine administration, we used the following linear model with interaction: accessibility $\approx$ TSS_enrichment + batch + $s e x+$ age + monocytes $+T_{-}$cells $+B_{-}$cells $+N K_{-}$cells $+N K T_{-} c e l l s+v i s i t+$ time + visit:time, where TSS_enrichment is a sample quality statistic defined in the previous section, batch refers to the experimental batch of the ATAC-seq library, sex and age refer to respective donor annotations, monocytes, T_cells, B_cells, NK_cells, and NKT_cells refer to cell proportions of the respective cell types estimated with Coulter counter (from PBMCs) and flow cytometry (from whole blood samples), visit is a categorical term encoded as 0,1 , and 2 for the first (baseline), second (2 weeks after BCG), and third (3 months after BCG) visit, respectively, and time is a categorical term encoded as 0 for morning (8 to $9 \mathrm{am}$ ) and 1 for evening ( 6 to $6: 30 \mathrm{pm}$ ) vaccination. We fit this model using R package LIMMA-voom (71) with TMM normalization (72) and duplicate correlation function with blocking on donor identity followed by empirical Bayes function for shrinking the variance across all tested peaks. We used false discovery rate (FDR) to correct for multiple testing across all tested peaks with the Benjamini-Hochberg method. We were interested in the regression coefficients and $P$ values of the visit:time interaction term. Given the aforementioned encoding of visit and time terms, positive coefficients identify regions where BCG-induced chromatin remodeling (second or third visit compared with first visit) results in a relative increase in accessibility if vaccinated in the evening compared with morning. We used R package LOLA (73) to identify enrichment of TF binding sites (TFBSs) in the open chromatin regions. To this end, we tested all peaks passing an FDR of 0.1, separately for peaks with positive and negative coefficients, against CODEX (74), a database of TFBSs determined with ChIP-seq in hematopoietic and embryonic cell lines, and JASPAR (75), a database of manually curated experimentally defined TF binding profiles. We used the BED files from LOLACore v180412 and LOLAExt v170206 for CODEX and JASPAR, respectively.

To identify enrichment of known molecular pathways, we mapped each peak to the nearest feature based on GENCODE (see previous 
section for details). Next, we retained only peaks that mapped to protein-coding features and were annotated as TSS, TSS_proximal, gene body, or distal but within a distance of 10,000 bp from the TSS. Finally, we tested peaks passing an FDR of 0.1, separately for peaks with positive and negative coefficients, using the online platform Enrichr (76), which performs Fisher's exact test, for enrichment of KEGG_2019 Human pathways (77).

Complete blood count. Complete blood counts were performed on EDTA whole blood and PBMC fractions after Ficoll isolation on a Sysmex XN-450 hematology analyzer.

Serum cortisol measurements. Serum cortisol was analyzed by LC-MS/MS after protein precipitation and solid-phase extraction as described previously (78), with the following additional compound-specific configurations and characteristics. $\left[{ }^{13} \mathrm{C}_{3}\right]$-cortisol (Isoscience) was used as the internal standard. Retention time was 1.46 minutes. A 9-point calibration curve was used (MilliporeSigma). Two transitions (qualitative and quantitative) were monitored. Transitions (Q1 $\rightarrow \mathrm{Q} 3)$ were $m / z 363.4 \rightarrow 121.1(25 \mathrm{kEV})$ and $m / z 363.4 \rightarrow 97.1$ (34 kEV) for cortisol; $m / z 366.4 \rightarrow 124.1$ (25 kEV) and $m / z 366.4 \rightarrow 100.1$ (35 kEV) for ${ }^{13} \mathrm{C}_{3}$-cortisol. Dwell time was $100 \mathrm{~ms}$. The method was assessed for linearity by the CLSI EP6 protocol. Recovery was within $96.5 \%$ to $102 \%$. Total CV for cortisol was $3.6 \%$ at $301 \mathrm{nmol} / \mathrm{L}$ and $3.1 \%$ at $1,092 \mathrm{nmol} / \mathrm{L}$. LOQ was 1.91 nmol/L (13.4\% CV).

Statistics. Raw cytokine values were first log transformed, and then corrected for batch effects using a linear regression model. These data conversions were performed using the statistical programming language R. $R^{2}$ represents the explained variance. Corrected cytokine values were converted to fold changes from baseline. Cytokine values were nonnormally distributed, as previously demonstrated in detail (79). A Mann-Whitney $U$ test was used to compare fold changes between morning- and evening-vaccinated groups. Kruskal-Wallis test with Dunn's multiple-comparison test was used to test for differences in the 4 different vaccinated subgroups divided by time of vaccination in the $300 \mathrm{BCG}$ cohort. Wilcoxon's matched-pairs signed-rank test was used to compare fold changes of in vitro-trained samples belonging to the same volunteer. Friedman's test with Dunn's multiple-comparison test was used to com- pare multiple groups of samples belonging to the same volunteer. Complete blood count values were converted to fold changes from baseline. A 2 -sided $P$ value of less than 0.05 was considered statistically significant.

Study approval. The 300BCG study was approved by the ArnhemNijmegen medical ethical committee (NL58553.091.16). The study was performed in accordance with the declaration of Helsinki. Written informed consent was obtained from the healthy volunteers during the first visit.

\section{Author contributions}

MGN and LCJDB designed the study. VPM, LCJDB, VACMK, SJCFMM, HL, and HD conducted the cohort study and performed the experiments. LCJDB and RJ performed the in vitro experiments. LCJDB, VPM, and VACMK analyzed the data. TK and VFG performed the ATAC-seq library preparation, DB processed the sequenced ATAC-seq samples, and LF performed the differential chromatin accessibility analysis. RJWA performed experiments and $\mathrm{BN}$ analyzed RNA-seq data from the previous study. MGN, RVC, LABJ, CB, and CSB supervised the analysis and interpretation of results. LCJDB and VPM wrote the manuscript, which was critically reviewed and approved by all authors. The order of co-first authorship of LCJDB and VPM was defined by the primary responsibility.

\section{Acknowledgments}

The authors would like to thank all volunteers for participating in the 300BCG cohort. We would also like to thank Trees Jansen for her assistance with labeling of all study materials, and Rob ter Horst for helpful discussions. MGN was supported by a Spinoza grant of the Netherlands Organization for Scientific Research and a European Research Council Advanced Grant (TRAIN-OLD no. 833247). LCJDB was partly funded by a grant to the Research Center for Vitamins and Vaccines (CVIVA) from the Danish National Research Foundation (DNRF108).

Address correspondence to: Mihai G. Netea, Radboudumc, Geert Grooteplein 8, 6525 GA Nijmegen, Netherlands. Phone: 31.24.366.18819; Email: mihai.netea@radboudumc.nl.
1. WHO. BCG immunization coverage estimates by WHO region. 2018. Global Health Observatory data repository. https://apps.who.int/gho/data/ view.main.81500?lang=en. Updated August 26, 2019. Accessed August 4, 2020.

2. Trunz BB, Fine P, Dye C. Effect of BCG vaccination on childhood tuberculous meningitis and miliary tuberculosis worldwide: a meta-analysis and assessment of cost-effectiveness. Lancet. 2006;367(9517):1173-1180.

3. Colditz GA, et al. Efficacy of BCG vaccine in the prevention of tuberculosis. Meta-analysis of the published literature. JAMA. 1994;271(9):698-702.

4. Brewer TF. Preventing tuberculosis with bacillus Calmette-Guérin vaccine: a meta-analysis of the literature. Clin Infect Dis. 2000;31 suppl 3:S64-S67.

5. Voss G, et al. Progress and challenges in TB vaccine development. F100ORes. 2018;7:199.

6. Kleinnijenhuis J, van Crevel R, Netea MG. Trained immunity: consequences for the heterologous effects of BCG vaccination. Trans $R$ Soc Trop Med Hyg. 2015;109(1):29-35.
7. Aaby P, et al. Randomized trial of BCG vaccination at birth to low-birth-weight children: beneficial nonspecific effects in the neonatal period? J Infect Dis. 2011;204(2):245-252.

8. Biering-Sørensen $S$, et al. Small randomized trial among low-birth-weight children receiving bacillus Calmette-Guérin vaccination at first health center contact. Pediatr Infect Dis J. 2012;31(3):306-308.

9. Biering-Sørensen S, et al. Early BCG-Denmark and neonatal mortality among infants weighing $<2500$ g: A randomized controlled trial. Clin Infect Dis. 2017;65(7):1183-1190.

10. Biering-Sørensen S, Jensen KJ, Monterio I, Ravn $\mathrm{H}$, Aaby P, Benn CS. Rapid protective effects of early BCG on neonatal mortality among low birth weight boys: observations from randomized trials. J Infect Dis. 2018;217(5):759-766.

11. Benn CS, Netea MG, Selin LK, Aaby P. A small jab - a big effect: nonspecific immunomodulation by vaccines. Trends Immunol. 2013;34(9):431-439.

12. de Bree LCJ, et al. Non-specific effects of vac- cines: Current evidence and potential implications. Semin Immunol. 2018;39:35-43.

13. Schaltz-Buchholzer F, et al. Early BCG vaccination, hospitalizations, and hospital deaths: analysis of a secondary outcome in 3 randomized trials from Guinea-Bissau. J Infect Dis. 2019;219(4):624-632.

14. Netea MG, Latz E, Mills KH, O’Neill LA. Innate immune memory: a paradigm shift in understanding host defense. Nat Immunol. 2015;16(7):675-679.

15. Kleinnijenhuis J, et al. BCG-induced trained immunity in NK cells: Role for non-specific protection to infection. Clin Immunol. 2014;155(2):213-219.

16. Kleinnijenhuis J, et al. Bacille Calmette-Guerin induces NOD2-dependent nonspecific protection from reinfection via epigenetic reprogramming of monocytes. Proc Natl Acad Sci US A. 2012;109(43):17537-17542.

17. Arts RJW, et al. Immunometabolic pathways in BCG-induced trained immunity. Cell Rep. 
2016;17(10):2562-2571.

18. Kaufmann E, et al. BCG educates hematopoietic stem cells to generate protective innate immunity against tuberculosis. Cell. 2018;172(1-2):176-190.e19.

19. Cirovic B, et al. BCG vaccination in humans elicits trained immunity via the hematopoietic progenitor compartment. Cell Host Microbe. 2020;28(2):322-334.e5.

20. Arts RJW, et al. BCG vaccination protects against experimental viral infection in humans through the induction of cytokines associated with trained immunity. Cell Host Microbe. 2018;23(1):89-100.e5.

21. Cheng SC, et al. mTOR- and HIF- $1 \alpha$-mediated aerobic glycolysis as metabolic basis for trained immunity. Science. 2014;345(6204):1250684.

22. Bekkering S, Blok BA, Joosten LA, Riksen NP, van Crevel R, Netea MG. In vitro experimental model of trained innate immunity in human primary monocytes. Clin Vaccine Immunol. 2016;23(12):926-933.

23. Walk J, et al. Outcomes of controlled human malaria infection after BCG vaccination. Nat Commun. 2019;10(1):874.

24. Boivin DB, James FO, Wu A, Cho-Park PF, Xiong $\mathrm{H}$, Sun ZS. Circadian clock genes oscillate in human peripheral blood mononuclear cells. Blood. 2003;102(12):4143-4145.

25. Adrover JM, et al. A neutrophil timer coordinates immune defense and vascular protection. Immunity. 2019;50(2):390-402.e10.

26. Keller M, et al. A circadian clock in macrophages controls inflammatory immune responses. Proc Natl Acad Sci U S A. 2009;106(50):21407-21412.

27. Curtis AM, Bellet MM, Sassone-Corsi P, O’Neill LA. Circadian clock proteins and immunity. Immunity. 2014;40(2):178-186.

28. Scheiermann C, Kunisaki Y, Frenette PS. Circadian control of the immune system. Nat Rev Immunol. 2013;13(3):190-198.

29. Labrecque N, Cermakian N. Circadian clocks in the immune system. J Biol Rhythms. 2015;30(4):277-290.

30. Long JE, Drayson MT, Taylor AE, Toellner KM, Lord JM, Phillips AC. Morning vaccination enhances antibody response over afternoon vaccination: A cluster-randomised trial. Vaccine. 2016;34(24):2679-2685.

31. Phillips AC, Gallagher S, Carroll D, Drayson M. Preliminary evidence that morning vaccination is associated with an enhanced antibody response in men. Psychophysiology. 2008;45(4):663-666.

32. Kurupati RK, et al. The effect of timing of influenza vaccination and sample collection on antibody titers and responses in the aged. Vaccine. 2017;35(30):3700-3708.

33. Stevens WB, Netea MG, Kater AP, van der Velden WJ. 'Trained immunity': consequences for lymphoid malignancies. Haematologica. 2016;101(12):1460-1468.

34. Mulder WJM, Ochando J, Joosten LAB, Fayad ZA, Netea MG. Therapeutic targeting of trained immunity. Nat Rev Drug Discov. 2019;18(7):553-566.

35. van Kerkhof LW, et al. Diurnal variation of hormonal and lipid biomarkers in a molecular epidemiology-like setting. PLoS One. 2015;10(8):e0135652.
36. Nissen TN, et al. Bacillus Calmette-Guérin vaccination at birth and in vitro cytokine responses to non-specific stimulation. A randomized clinical trial. Eur J Clin Microbiol Infect Dis. 2018;37(1):29-41.

37. Early JO, Curtis AM. Immunometabolism: Is it under the eye of the clock? Semin Immunol. 2016;28(5):478-490.

38. Man K, Loudon A, Chawla A. Immunity around the clock. Science. 2016;354(6315):999-1003.

39. Bureau JP, Garrelly L, Vago P. Nycthemeral variations on LPS- and BCG-induced PMN migration in normal mice. Int J Tissue React. 1991;13(4):203-206.

40. Bureau JP, Labrecque G, Coupé M, Garrelly L. Influence of BCG administration time on the in-vivo migration of leukocytes. Chronobiol Int. 1986;3(1):23-28.

41. Lopes C, Mariano M, Markus RP. Interaction between the adrenal and the pineal gland in chronic experimental inflammation induced by BCG in mice. Inflamm Res. 2001;50(1):6-11.

42. Tsai TH, Burns RE, Scheving LE. Circadian influence on the immunization of mice with live bacillus Calmette-Guérin (BCG) and subsequent challenge with Ehrlich ascites carcinoma. Chronobiologia. 1979;6(3):187-201.

43. Kiessling S, Dubeau-Laramée G, Ohm H, Labrecque N, Olivier M, Cermakian N. The circadian clock in immune cells controls the magnitude of Leishmania parasite infection. Sci Rep. 2017;7(1):10892.

44. Dos Santos JC, et al. $\beta$-Glucan-induced trained immunity protects against Leishmania braziliensis Infection: a crucial role for IL-32. Cell Rep. 2019;28(10):2659-2672.e6.

45. Tognini P, Thaiss CA, Elinav E, Sassone-Corsi P. Circadian coordination of antimicrobial responses. Cell Host Microbe. 2017;22(2):185-192.

46. Astrinidis A, et al. The transcription factor SP1 regulates centriole function and chromosomal stability through a functional interaction with the mammalian target of rapamycin/raptor complex. Genes Chromosomes Cancer. 2010;49(3):282-297.

47. Jablonska A, Polouliakh N. In silico discovery of novel transcription factors regulated by mTOR-pathway activities. Front Cell Dev Biol. 2014;2:23.

48. Yamada T, Gierach K, Lee PH, Wang X, Lacorazza HD. Cutting edge: Expression of the transcription factor E74-like factor 4 is regulated by the mammalian target of rapamycin pathway in CD8 ${ }^{+}$T cells. JImmunol. 2010;185(7):3824-3828.

49. Wang Y, et al. Krüppel-like factor 4 is induced by rapamycin and mediates the anti-proliferative effect of rapamycin in rat carotid arteries after balloon injury. Br J Pharmacol. 2012;165(7):2378-2388.

50. Rijo-Ferreira F, Takahashi JS. Genomics of circadian rhythms in health and disease. Genome Med. 2019;11(1):82.

51. Edgar RS, et al. Cell autonomous regulation of herpes and influenza virus infection by the circadian clock. Proc Natl Acad Sci U S A. 2016;113(36):10085-10090.

52. Majumdar T, Dhar J, Patel S, Kondratov R, Barik S. Circadian transcription factor BMAL1 regulates innate immunity against select RNA viruses. Innate Immun. 2017;23(2):147-154.
53. Abadie V, et al. Neutrophils rapidly migrate via lymphatics after Mycobacterium bovis BCG intradermal vaccination and shuttle live bacilli to the draining lymph nodes. Blood. 2005;106(5):1843-1850.

54. Duffy D, et al. Neutrophils transport antigen from the dermis to the bone marrow, initiating a source of memory $\mathrm{CD}^{+} \mathrm{T}$ cells. Immunity. 2012;37(5):917-929.

55. Méndez-Ferrer S, Lucas D, Battista M, Frenette PS. Haematopoietic stem cell release is regulated by circadian oscillations. Nature. 2008;452(7186):442-447.

56. García-García A, et al. Dual cholinergic signals regulate daily migration of hematopoietic stem cells and leukocytes. Blood. 2019;133(3):224-236.

57. Nagai Y, et al. Toll-like receptors on hematopoietic progenitor cells stimulate innate immune system replenishment. Immunity. 2006;24(6):801-812.

58. Takizawa H, Boettcher S, Manz MG. Demand-adapted regulation of early hematopoiesis in infection and inflammation. Blood. 2012;119(13):2991-3002.

59. Early JO, et al. Circadian clock protein BMAL1 regulates IL-1 $\beta$ in macrophages via NRF2. Proc Natl Acad Sci U S A. 2018;115(36):E8460-E8468.

60. Coutinho AE, Chapman KE. The anti-inflammatory and immunosuppressive effects of glucocorticoids, recent developments and mechanistic insights. Mol Cell Endocrinol. 2011;335(1):2-13.

61. Nemes E, et al. Prevention of M. tuberculosis infection with H4:IC31 vaccine or BCG revaccination. N Engl JMed. 2018;379(2):138-149.

62. Buenrostro JD, Giresi PG, Zaba LC, Chang HY, Greenleaf WJ. Transposition of native chromatin for fast and sensitive epigenomic profiling of open chromatin, DNA-binding proteins and nucleosome position. Nat Methods. 2013;10(12):1213-1218.

63. Corces MR, et al. Lineage-specific and single-cell chromatin accessibility charts human hematopoiesis and leukemia evolution. Nat Genet. 2016;48(10):1193-1203.

64. Jiang H, Lei R, Ding SW, Zhu S. Skewer: a fast and accurate adapter trimmer for next-generation sequencing paired-end reads. BMC Bioinformatics. 2014;15:182.

65. Langmead B, Salzberg SL. Fast gapped-read alignment with Bowtie 2. Nat Methods. 2012;9(4):357-359.

66. Tarasov A, Vilella AJ, Cuppen E, Nijman IJ, Prins P. Sambamba: fast processing of NGS alignment formats. Bioinformatics. 2015;31(12):2032-2034.

67. Zhang Y, et al. Model-based analysis of ChIP-Seq (MACS). Genome Biol. 2008;9(9):R137.

68. ENCODE Project Consortium. An integrated encyclopedia of DNA elements in the human genome. Nature. 2012;489(7414):57-74.

69. Frankish A, et al. GENCODE reference annotation for the human and mouse genomes. Nucleic Acids Res. 2019;47(D1):D766-D773.

70. Kondili M, Fust A, Preussner J, Kuenne C, Braun T, Looso M. UROPA: a tool for Universal RObust Peak Annotation. Sci Rep. 2017;7(1):2593.

71. Law CW, Chen Y, Shi W, Smyth GK. voom: Precision weights unlock linear model analysis tools for RNA-seq read counts. Genome Biol. 
2014;15(2):R29.

72. Robinson MD, Oshlack A. A scaling normalization method for differential expression analysis of RNA-seq data. Genome Biol. 2010;11(3):R25.

73. Sheffield NC, Bock C. LOLA: enrichment analysis for genomic region sets and regulatory elements in R and Bioconductor. Bioinformatics. 2016;32(4):587-589.

74. Sánchez-Castillo M, et al. CODEX: a nextgeneration sequencing experiment database for the haematopoietic and embryonic stem cell communities. Nucleic Acids Res. 2015;43(Database issue):D1117-D1123.

75. Fornes O, et al. JASPAR 2020: update of the open-access database of transcription factor binding profiles. Nucleic Acids Res. 2020;48(D1):D87-D92.

76. Kuleshov MV, et al. Enrichr: a comprehensive gene set enrichment analysis web server 2016 update. Nucleic Acids Res. 2016;44(W1):W90-W97.
77. Kanehisa M, Furumichi M, Tanabe M, Sato Y, Morishima K. KEGG: new perspectives on genomes, pathways, diseases and drugs. Nucleic Acids Res. 2017;45(D1):D353-D361.

78. Ter Horst R, et al. Host and environmental factors influencing individual human cytokine responses. Cell. 2016;167(4):1111-1124.e13.

79. Li Y, et al. Inter-individual variability and genetic influences on cytokine responses to bacteria and fungi. Nat Med. 2016;22(8):952-960. 